\title{
The turbulent jet from a slender annular slot ventilated by a self-induced flow through the open core
}

\author{
Shahid A. Padhani, ${ }^{1}$ Gary R. Hunt,${ }^{1, *}$ and Timothy N. Jukes ${ }^{2}$ \\ ${ }^{1}$ Department of Engineering, University of Cambridge, \\ Cambridge, CB2 1PZ, UK \\ ${ }^{2}$ Aero-Acoustic Research, Dyson Technology Ltd. \\ Malmesbury, Wiltshire, SN16 ORP, UK
}

(Dated: December 27, 2017)

\begin{abstract}
The dynamics of an incompressible turbulent air jet from an annular source into otherwise quiescent surroundings are investigated. Focussing on a slender annulus with an open core, the development of the jet is examined using planar and stereoscopic particle image velocimetry (PIV) from the source to 16 (outer) source diameters downstream. Unique to these annular sources, the jet induces a flow through the open core - in other words, the core is 'ventilated'. Our measurements indicate that the volume flux drawn through the core exceeds that from the source by approximately $20 \%$. Based on the streamwise development of the jet, we identify four distinct regions: (i) an internal region of induced flow that is bounded by the jet; (ii) a near-field planar-jet-like region; (iii) a transition region; and, ultimately, (iv) a far-field round-jet-like region. We explore the evolution of the jet towards self-similar behaviour based on cross-stream profiles of time-averaged velocity and turbulence statistics within these different regions. Four distinct length scales are shown to characterise the streamwise extents and behaviours within the regions identified. Finally, to highlight the role of the open-core (ventilated) annular geometry on jet development, comparisons are made with PIV measurements of turbulent jets issuing from circular sources. These comparisons reveal that the ventilated geometry significantly enhances dilution in the near field.
\end{abstract}

Usage: Secondary publications and information retrieval purposes.

PACS numbers: May be entered using the \pacs\{\#1\} command.

\section{INTRODUCTION}

\section{A. Problem Outline}

The flow of interest herein is that established by an incompressible, turbulent and isothermal jet that issues from an annular source into otherwise quiescent surroundings. The source geometry of interest is depicted schematically in Fig. 1(a) and comprises an annular slot, from which the source fluid issues, circumnavigating an open core through which fluid may pass. Our focus is on steady turbulent releases from slots whose outer diameter $\left(D_{o}\right)$ only marginally exceeds the inner diameter $\left(D_{i}\right)$, i.e. slender annuli for which $D_{o}-D_{i} \ll D_{i}$, or equivalently, those with a diameter ratio $D_{i} / D_{o} \approx 1$. As we show in Sec. III, uniquely, the jet which develops from this source geometry (herein we adopt $D_{i} / D_{o}=0.968$ ) induces a flow in the ambient that passes through the open core in the direction of the jet. In other words, the open core may be regarded as being 'ventilated' by this induced flow (Fig. 1b). These features are clearly observed in the flow visualisation of the annular jet in Fig. 2. The current work is intentionally limited to a single value of $D_{i} / D_{o}(=0.968)$, which was as close to the limiting slender case $\left(D_{i} / D_{o}=1\right)$ as was feasibly possible with the fabrication technique. In a separate study [1], the role of $D_{i} / D_{o}$ was examined, and crucially, for these slender annuli the overall structure and behaviour remained unchanged.

\footnotetext{
* gary.hunt@eng.cam.ac.uk
} 


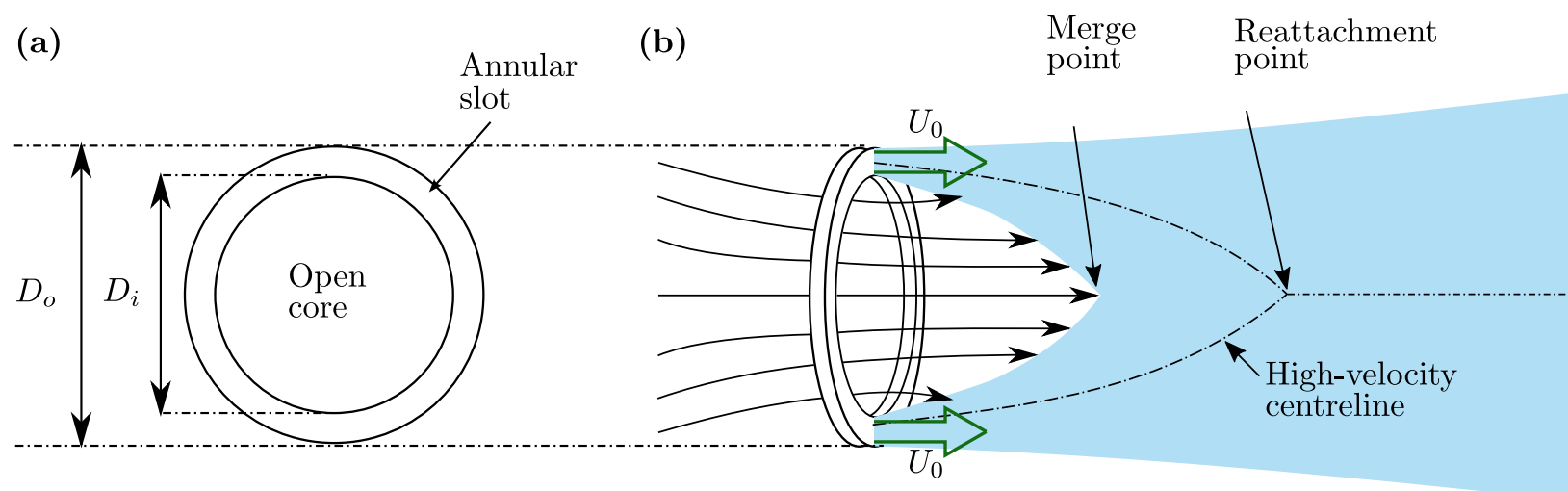

FIG. 1: Schematics depicting: (a) an open-core annular-jet source, shown face on to the exit slot of width $l=\left(D_{o}-D_{i}\right) / 2$; (b) time-averaged streamwise section through the jet showing streamlines for the ventilating flow induced through the core of the annulus. The shaded region depicts the main body of the jet. The steady exit velocity $U_{0}$ is perpendicular to the plane of the source as indicated. Relative locations of 'merge' and 'reattachment' points (defined in Sec. III) are indicated.

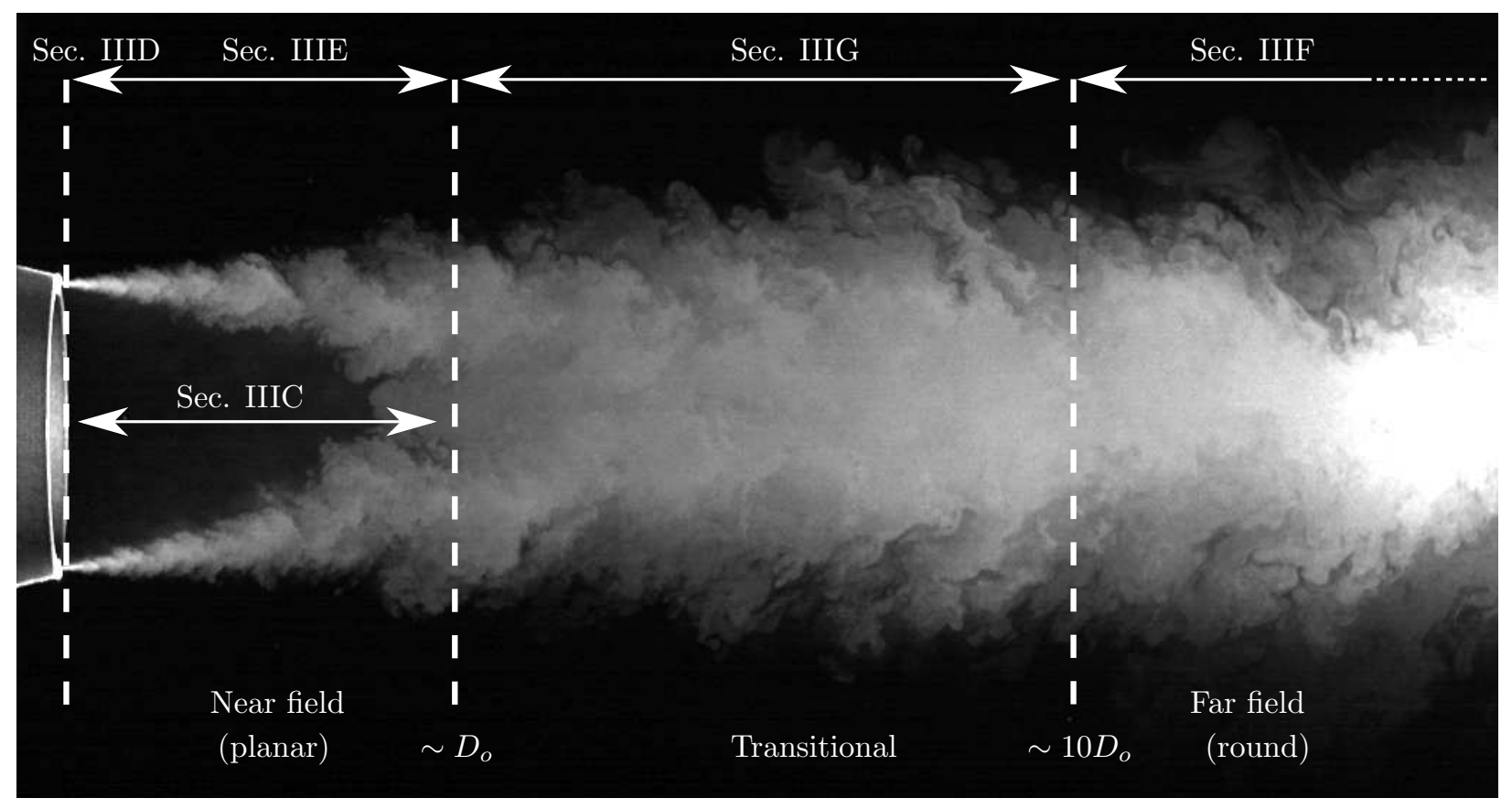

FIG. 2: Flow visualisation of a ventilated annular air jet from an open-core prototype slender annular source $\left(D_{i} / D_{o}=0.981\right.$ and Reynolds number based on the slot width of $\left.R e_{l} \approx 1800\right)$.

The flow was visualised using vaporised olive oil particles fed directly into the intake of the supply pump. The image, taken of the instantaneous flow in the mid-plane of the jet, clearly shows a near-field region of jet coalescence that bounds an internal region of induced flow that is drawn through the open core in the direction of the jet (left to right). The jet is partitioned (not to scale) into the distinct regions of flow identified (see Secs. III B-III F). 
Due to their wide application in combustion and mixing processes, the existing literature reports extensively on the fluid mechanics of annular jets that issue from closed-core, i.e. unventilated, sources [e.g. 2-11]. By contrast, the development of jets from open-core slender $\left(D_{i} / D_{o} \approx 1\right)$ annular sources has been entirely neglected until now. As such, we believe the findings presented herein to be the first on this class of jet. A review of the literature pertinent to the ventilated annular source is given in Sec. IB. Despite the fluid mechanics of these jets not receiving attention until now, devices intended for personalised cooling $[12,13]$ that produce air jets from open-core ventilated annular sources are widely available commercially and are commonplace in many homes and offices at the time of writing. Whilst we are not aware of other applications, our results (Sec. III) suggest that the enhanced near-field dilution that is achieved by the open-core annular source geometry (relative to that achieved by a classic round jet from a circular source) may lend itself to wider applications, for example, those concerning pollutant discharges where rapid near-nozzle dilution of the source fluid may be desirable.

\section{B. Background \& context}

\section{Unventilated annular jets}

The main features of unventilated (i.e. closed-core) annular jets were captured by the experimental investigations in air of Chigier and Beer [14], Ko and Chan [15, 16] and Chan and Ko [17]. Flow from the source was observed to self-entrain and subsequently to coalesce downstream, leading to the formation of a large-scale unsteady recirculation region adjacent to the source from which large-scale turbulent structures, referred to as wake vortices, were shed. Further downstream, Chan and Ko [17] recorded a linear growth in jet width and an inverse linear decay in streamwise velocity - their results thereby affirming that the unventilated annular jet behaves similarly to a classic round jet [cf. 18] in the far field.

The source conditions of an unventilated annular jet are conventionally characterised by a specific momentum flux $M_{0}\left(\propto l\left(D_{o}+D_{i}\right) U_{0}^{2}\right)$ and a Reynolds number $R e_{l}$ based on the slot width $l=\left(D_{o}-D_{i}\right) / 2$

$$
R e_{l}=\frac{U_{0} l}{v}
$$

$[14,15]$ where $U_{0}$ denotes the exit velocity, and $v$ the kinematic viscosity of the source fluid and environment. Using source geometries with diameter ratios in the range $0.56<D_{i} / D_{o}<0.85, \mathrm{Li}$ and Tankin [19] observed that the scale of the recirculation region and the jet envelope became independent of Reynolds number for $R e_{l}>800$.

\section{Ventilated annular jets}

To our knowledge, there exists only one previous study in which the fluid mechanics of a jet from a ventilated annular source is examined, namely that of Warda et al. [20]. They considered a source with $D_{i} / D_{o}=0.66$, i.e. a non-slender geometry relative to that considered herein, and while their source can be considered to be ventilated, its geometry is quite distinct from that which forms our focus. Most notably, the design of their nozzle was such that the diameter of the central opening was significantly less than the inner diameter $D_{i}$ and located upstream of the jet exit plane (rather than on this plane as in Fig. 1); additionally, a circular baffle was located downstream of their central opening. Crucially, the restricted through-nozzle ventilation afforded by their small central opening and baffle resulted in the formation of a large-scale recirculation region, similar 
to that observed for unventilated annular jets. Despite the similarities with unventilated annular jets, Warda et al. [20] determined that ventilation of the core results in the jet reattaching (Fig. 1b) further downstream than for the unventilated case [cf. 15]. To be consistent with the existing terminology on unventilated annular jets, herein we refer to the merging of the jet centreline as 'reattachment'. Warda et al. [20] explain this behaviour on reasoning that the flow through the core leads to a reduction in the pressure difference between the recirculation region and the ambient. Finally, their laser Doppler anemometry measurements confirm that sufficiently far downstream of the nozzle (beyond $7.8 D_{o}$ ) the jet tends toward the well-established round-jet behaviour. As we shall see in Sec. III, no such large-scale recirculation is present for the slender ventilated annular source geometry $D_{i} / D_{o} \approx 1$.

Due to the geometrical similarities shared by annular and coaxial-jet nozzles, the latter referring to a central circular opening surrounded by an annular opening, the source conditions of interest herein may be regarded as a special case of a coaxial jet. Specifically, a coaxial jet in which the exit velocity of the outer jet is imposed $\left(\right.$ as $\left.U_{0}\right)$ and the 'exit' velocity of the inner jet is induced naturally as a result of entrainment into the outer jet (rather than imposed as is the norm). From the research of Rehab et al. [21] on coaxial jets, it is clear that the flow rate through the central core will control the existence and scale of a recirculating region; for a sufficiently large flow rate through the core, a recirculation region will not exist and as the flow rate is reduced, a recirculation region will form and grow in scale. If the velocity through the central opening is set to zero, the coaxial jet resembles an unventilated annular jet, producing a large-scale recirculation region. However, despite these geometrical similarities, the flow rate through the core of a general ventilated annular jet is not controlled directly but rather by the slot geometry and slot fluxes; the presence and size of a recirculation region are a consequence of the jets ability to induce a flow through the core.

\section{Layout of this article}

Based on a review of the literature it is evident that the turbulent jet which forms from an opencore slender annular source has not been considered prior to the current work. To advance our understanding we have explored using PIV the streamwise and cross-stream development of such a jet, from the region immediately adjacent to the source to $16 D_{o}$ downstream. Our results provide entirely new information on the annular jet. We proceed (Sec. II) by outlining the experimental set-up designed specifically for this purpose and the procedures used to obtain our measurements. This is followed by our results (Sec. III) in which we utilise the PIV data to examine the streamwise variation of the momentum integral. We then examine the development towards self-similar behaviour and, in doing so, identify key locations of dynamical interest, e.g. where the jet merges and reattaches. The induced-flow region is also investigated, including a quantification of the volume flux induced through the central core. In Sec. IV we summarise our findings and draw our conclusions.

\section{EXPERIMENTS}

A programme of experiments was conducted in which instantaneous velocity measurements of an open-core annular air jet were obtained using PIV. Details of our experimental set-up, including the nozzle design, the source conditions and the diagnostics used are given below. 
TABLE I: Nozzle dimensions and source conditions. Reynolds numbers, $R e_{l}=U_{0} l / v(1)$ and

$R e_{f}=\sqrt{M_{0}} / v(2)$, are those estimated based on a kinematic viscosity for air at $20^{\circ} \mathrm{C}$ of $v=1.5 \times 10^{-5} \mathrm{~m}^{2} \mathrm{~s}^{-1}[22]$ and on assuming a uniform exit velocity $U_{0}=Q_{0} / A_{0} ; Q_{0}=0.0153$ $\mathrm{m}^{3} \mathrm{~s}^{-1} \pm 1 \%$ denotes the source volume flux and $A_{0}=\pi\left(D_{o}^{2}-D_{i}^{2}\right) / 4=5.184 \mathrm{~cm}^{2}$ the area of the nozzle exit. The slot width was measured using digital vernier calipers to $\pm 0.001 \mathrm{~cm}$ and varied by $\pm 5 \%$ around the circumference of the annulus.

\begin{tabular}{llllllllll}
\hline \hline $\begin{array}{l}D_{o} \\
(\mathrm{~cm})\end{array}$ & $\begin{array}{l}D_{i} \\
(\mathrm{~cm})\end{array}$ & $\frac{D_{i}}{D_{o}}$ & $\begin{array}{l}l \\
(\mathrm{~cm})\end{array}$ & $\begin{array}{l}h \\
(\mathrm{~cm})\end{array}$ & $\begin{array}{l}A_{0} \\
\left(\mathrm{~cm}^{2}\right)\end{array}$ & $\begin{array}{l}\sqrt{A_{0}} \\
(\mathrm{~cm})\end{array}$ & $\begin{array}{l}U_{0} \\
\left(\mathrm{~m} \mathrm{~s}^{-1}\right)\end{array}$ & $R e_{l}$ & $R e_{f}$ \\
\hline 10.165 & 9.835 & 0.968 & 0.165 & 0.8 & 5.184 & 2.277 & 29.5 & $3200 \pm 5 \%$ & $45000 \pm 5 \%$ \\
\hline \hline
\end{tabular}

\section{A. Nozzle design \& source conditions}

Experiments were performed in a test chamber of horizontal dimensions $5 \mathrm{~m} \times 4 \mathrm{~m}$ and height $3 \mathrm{~m}$ using a stereolithography (SLA) 3D printed annular nozzle made of accura25 polyamide. A slot width as fine as $l=\left(D_{o}-D_{i}\right) / 2=1.65 \mathrm{~mm} \pm 5 \%$ could reliably be obtained with this method of fabrication. To enable a study of the key flow features in both the near and far fields of the jet, we converged on a nozzle with a mean diameter of $D_{m}=\left(D_{o}+D_{i}\right) / 2=10 \mathrm{~cm}$. We thereby achieved a near ideal diameter ratio of $D_{i} / D_{o}=0.968$, i.e. with $D_{i} / D_{o} \approx 1$ as desired for a slender annulus. For an idealised 'fully open' core annular source, the diameter of the central opening $D_{v}$ and the inner diameter of the slot are equal, giving a 'ventilation ratio' of $D_{v} / D_{i}=1$. The ventilation ratio of the nozzle used herein is limited by the constraints of the experiment and fabrication technique, thus the core of the annulus is marginally restricted by the nozzle itself with $D_{v} / D_{i} \approx 0.9$. A schematic of the nozzle fabricated is shown in Fig. 3 (dimensions are given in Table I). Seeking to achieve uniform release conditions at the exit plane of the slot, four air supply ports were located equidistant around the circumference of the nozzle (Fig. 3b), and the interior of the nozzle (of width $h=8 \mathrm{~mm}$ ) was lined with a $2 \mathrm{~cm}$ thick strip of porous foam. The nozzle had an area contraction ratio of 5:1 (approx.). Moreover, 16 vanes were fabricated within the nozzle, one vane every $22.5^{\circ}$, in order to provide structure and to channel the air in a direction perpendicular to the exit plane, thereby reducing swirl. With this design, our streamwise planar PIV measurements (taken immediately downstream, at $x / l=0.2$, of the exit plane) confirmed that the flow exiting the nozzle has an approximately uniform velocity profile with a turbulence intensity of approximately 5\%. This velocity profile can be seen in Fig. 11(a). Further details of the source conditions and near-source flow achieved with this design are given in Sec. III C. The nozzle was supported by threaded rods attached to the support connectors. These rods, arranged in an X-shape, were themselves held rigidly by a combination of retort stands and clamps.

Despite the use of similar source velocities, our slot Reynolds number of $R e_{l} \approx 3200$ is lower than for the unventilated annular jet studies of others, e.g. $R e_{l}=6.8 \times 10^{4}$ in the experiments of Ko and Chan [15]. This is due to the significantly narrower slot width of our nozzle that enabled us to achieve $D_{i} / D_{o} \approx 1$ and to explore both the near and far field of the jet in the test chamber. In their study of unventilated annular jets, Li and Tankin [19] observed turbulent flow for $R e_{l}>800$ and that the influence of further increases in $R e_{l}$ on the jet envelope was small; we might reasonably anticipate similar findings for ventilated annular jets. Indeed, using vaporised olive oil particles fed directly into the intake of the pump for flow visualisation, across a range of Reynolds numbers $\left(500<R e_{l}<2500\right)$ on a prototype ventilated slender annular nozzle $\left(D_{i} / D_{o}=0.984\right)$, confirmed that the jet envelope did not vary significantly for $R e_{l} \gtrsim 800$. The Reynolds number of 
(a) Cross-section (b) Front view

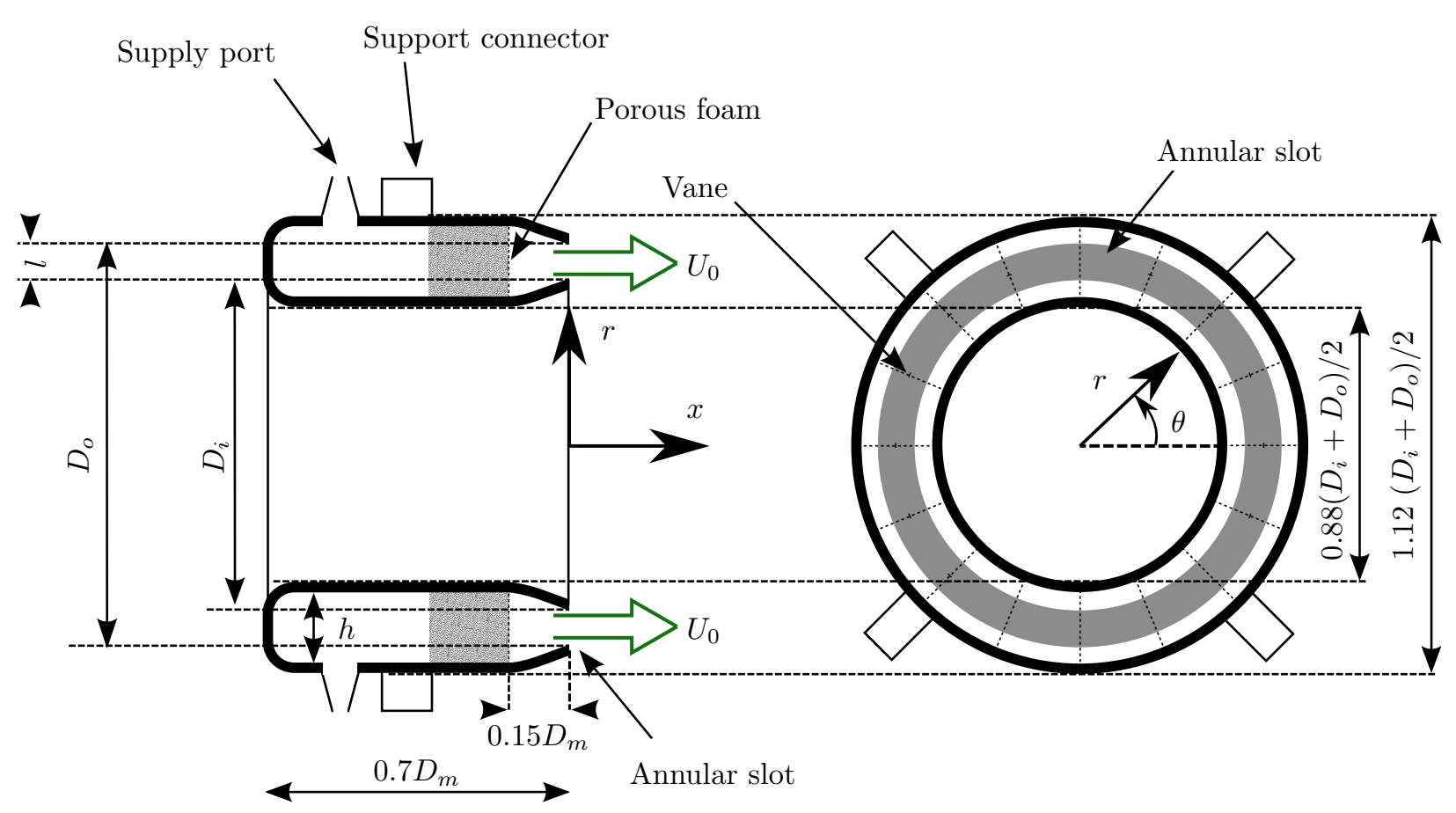

FIG. 3: Schematics showing the annular nozzle design (not to scale). The wall thickness (thick solid line) is approximately $2 \mathrm{~mm}$. (a) Section through the nozzle that bisects the air supply ports and the support connectors. Air pumped to the supply ports issues from the annular slot with mean velocity $U_{0}\left(\mathrm{~m} \mathrm{~s}^{-1}\right)$. (b) Front view showing the swirl-reducing vanes positioned at $22.5^{\circ}$ intervals. Nozzle dimensions are given in Table I. The coordinate system $(x, r, \theta)$ is marked.

the coalesced (far-field) jet based on the specific momentum flux of the source,

$$
\operatorname{Re}_{f}=\frac{Q_{0}}{\sqrt{A_{0}} v}=\frac{\sqrt{M_{0}}}{v}
$$

is estimated to be an order of magnitude larger than $R e_{l}$ at $R e_{f} \approx 45000$; in (2), $Q_{0}$ denotes the source volume flux and $A_{0}\left(=\pi\left(D_{o}^{2}-D_{i}^{2}\right) / 4\right)$ the slot area. Dimotakis [23] argues that fully developed turbulence in jets, and quasi-independence of the flow from the Reynolds number, require a Reynolds number based on the length scale of the nozzle to exceed $1-2 \times 10^{4}$. In the region near the nozzle, where the dominant length scale is the slot width $l$ ((1) and Sec. IIID), $R e_{l}=$ $3200 \ll 1-2 \times 10^{4}$ indicates that the influence of the Reynolds number may be significant. Further downstream however, where the dominant length scale is $\sqrt{A_{0}}$ (Sec. IIIE), the relatively large Reynolds number achieved herein, $R e_{f}=45000\left(>1-2 \times 10^{4}\right)$, indicates that the aspects of the flow we are most interested in will be insensitive to $R e_{f}$. The exit velocity of $U_{0}=29.5 \mathrm{~m} \mathrm{~s}^{-1}$ (Table I) results in a Mach number of $U_{0} / c \approx 0.09$ (taking the speed of sound in air as $c=343.4$ $\mathrm{m} \mathrm{s}^{-1}$, Haynes [24]), indicating that the jet may be considered to be incompressible.

The mechanism providing the controlled supply of air to the ports (described in Sec. II B) resulted in a temperature difference of $\Delta T=15^{\circ} \mathrm{C}$ between the jet and the ambient (as recorded using a RS51 digital thermocouple at the exit plane of the slot and in the ambient at the height 


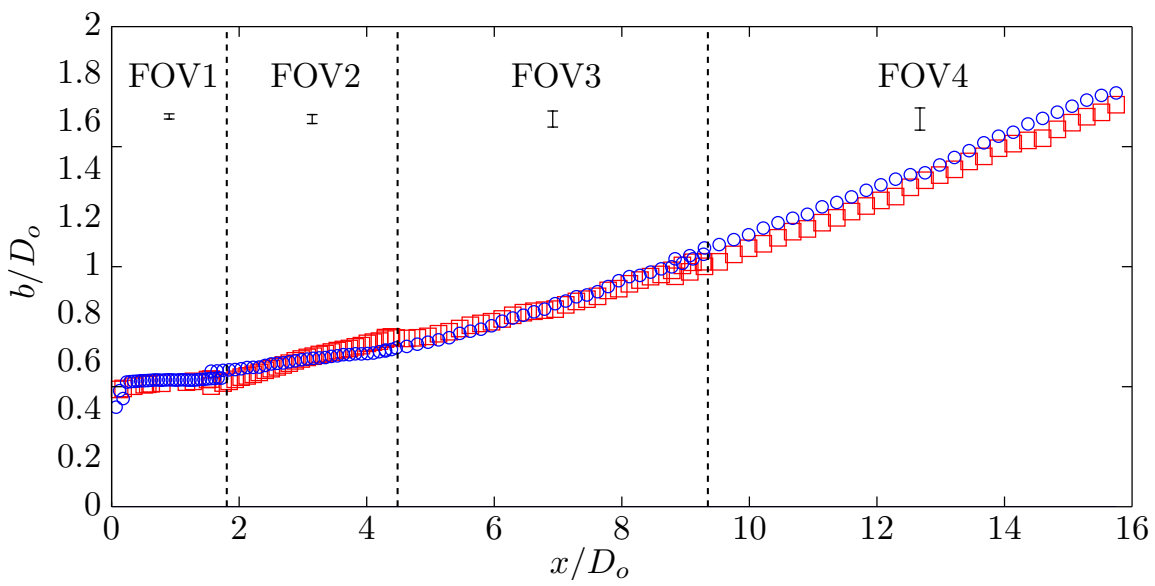

FIG. 4: A plot showing the width of the 'lower half' (o) and 'upper half' ( $\square$ ) of the shear layer in our slender open-core annular jet. The vertical dashed lines delineate the plot into four regions representing FOV1-FOV4, and the error bars represent the resolution of the measurements within each FOV.

of the nozzle). As a consequence, the jet was not perfectly isothermal due to a non-zero source buoyancy flux $B_{0}\left(=g Q_{0} \Delta T / T_{0}\right.$, where $g$ is the acceleration due to gravity and $T_{0}$ the temperature of the ambient).

To demonstrate that the source buoyancy flux does not influence our measurements of the jet, in figure 4 we compare the width of the 'lower half' and 'upper half' of the shear layer, as measured along a section which vertically intersects the mid-plane of the jet. Given that our jet issues parallel to the floor, if the influence of buoyancy was non negligible we would expect to see differences in the growth rates of the two halves of the shear layer. While some systematic differences are observed far from the nozzle, these can be attributed to the resolution of the measurements. The growth rate of the two halves does not appear to differ and thus we can conclude that the influence of buoyancy is small within our measurement domain. This is supported by measurements of the jet length $L_{j}=M_{0}^{3 / 4} B_{0}^{-1 / 2}$ [25], which provides us with a measure of the distance from the source over which the dynamics of the jet are not expected to be influenced by this temperature anomaly. Based on our source conditions $L_{j} \approx 6.5 \mathrm{~m}$ and, crucially, all our measurements were recorded within $0.25 L_{j}$ of the source, thereby confirming that the effect of buoyancy on our results may be regarded as negligible.

\section{B. Experimental set-up}

The test chamber was seeded using a TSI 9307-6 Laskin nozzle oil droplet generator (with diethyl-hexyl-sebacat oil) which produced particles with a diameter of approximately one micron. This seeded ambient air was fed to the nozzle, via a four-way manifold, by a mixed flow impeller fan controlled by a variable autotransformer (Variac).

Measurements of the pressure difference $\Delta P$ between the ambient and ducting downstream of the bell mouth were made using a Digitron 2020P7 $2.5 \mathrm{kPa}$ digital manometer. The reference background pressure was measured using an in-house barometer. The ambient temperature $T_{0}$ in the room was measured using an ATP DT-321 temperature and humidity meter. These measurements, combined with the measurements of $\Delta T$, allowed us to: estimate the source volume flux from application of Bernoulli's theorem; apply corrections based on the bell mouth calibration; 
and account for the expansion of air passing through the fan (due to the temperature difference $\Delta T)$. These steps resulted in a final estimate of $Q_{0}=0.0153 \mathrm{~m}^{3} \mathrm{~s}^{-1} \pm 1 \%$. Pressure fluctuations during a given experiment were within $\pm 5 \mathrm{~kg} \mathrm{~m}^{-1} \mathrm{~s}^{-2}$ (i.e. within $\pm 5 \mathrm{~Pa}$ ), indicating a variation in flow rate of $\pm 0.0001 \mathrm{~m}^{3} \mathrm{~s}^{-1}$. To ensure uniform conditions within the test chamber, the air was completely purged and replaced with external air prior to each experimental run. The air in the chamber was then allowed to settle prior to activating the jet and commencing an experiment. An examination of the axial variation of the momentum integral (Sec. III A) indicates that the return flow in the test chamber had a negligible effect on the jet.

The jet was illuminated using a vertical laser sheet of approximately $1 \mathrm{~mm}$ width, which was produced by a $532 \mathrm{~nm}$ Nd:YAG laser (Litron Lasers Nano L 200-15 PIV twin cavity) with sheet forming optics. The two cavities of the laser permitted two $10 \mathrm{~ns}$ pulses in rapid succession; each cavity had a maximum pulse rate of $15 \mathrm{~Hz}$, with a power output of $200 \mathrm{~mJ}$ per pulse. Images of the flow were captured using a 5.5 mega-pixel Imager sCMOS camera fitted with a Nikon $50 \mathrm{~mm}$ lens at $\mathrm{f} / 4$ and a $532 \mathrm{~nm}$ narrowband filter. The filter ensured the camera only received light from the laser. The camera and laser were synchronised using a programmable timing unit with a "double frame double exposure" method [26]. This recording method results in the two sequential laser pulses being recorded on two sequential frames. The duration between successive laser pulses was set so as to ensure a maximum particle displacement of approximately 7 pixels between successive images.

The majority of measurements recorded (Sec. III) were from planar PIV in which the camera was positioned perpendicular to the laser sheet that was aligned along the symmetry axis of the flow; data were thereby captured on the $x-y$ plane, where the $y$ axis corresponds to $r(\theta=\pi / 2)$. To capture the behaviour of the jet, both far from the source and in the near field whilst maintaining accuracy, four overlapping windows of increasing size were used. This arrangement, depicted in Fig. 5, enabled the time-averaged data collected to be combined into a single dataset spanning the entire measurement domain but with the penalty that the spatial resolution of the data decreased with increasing window size (details given in Table II). An additional planar PIV measurement window (referred to as NE 'nozzle exit'), captured using a Tokina $100 \mathrm{~mm}$ macro lens at f/8, was situated immediately downstream of the slot in order to examine the flow at the slot exit. A set of cross-sectional stereoscopic PIV measurements was also taken, with the vertical laser sheet aligned perpendicular to the longitudinal nozzle axis at $x=0.5 D_{o}$. For these measurements, two cameras were mounted $56 \mathrm{~cm}$ downstream of the measurement plane and on either side of the jet, with an angle of $36^{\circ}$ between the longitudinal nozzle axis and the optical axes of the cameras.

Calibration of the PIV system was performed using the DaVis 8.2.1 calibration tutorial, employing a camera pinhole model and the LaVison two-level calibration plate (type 309-15) of dimensions $0.309 \mathrm{~m} \times 0.309 \mathrm{~m}$. The self-calibration procedure was iterated several times when performing stereoscopic PIV. Prior to data acquisition, checks were made using the DaVis software to ensure strong correlation peaks and an absence of peak locking. Visual inspection revealed that spurious vectors, of which there were very few, were primarily situated at the corners of the domain, i.e. away from the region of interest. The effect that a sample of these spurious vectors had on our results is observed as outliers of turbulent intensity values in Fig. 16 - see the profile at $x / D_{o}=10$ for $y / b \lesssim-2.5$.

PIV measurements on a round jet produced by a nozzle with identical geometry and source conditions to the jet studied by Hussein et al. [18] were used to validate our PIV measurement system as discussed in Appendix A. These results for the round jet are compared with the ventilated annular jet measurements in Sec. III E. 


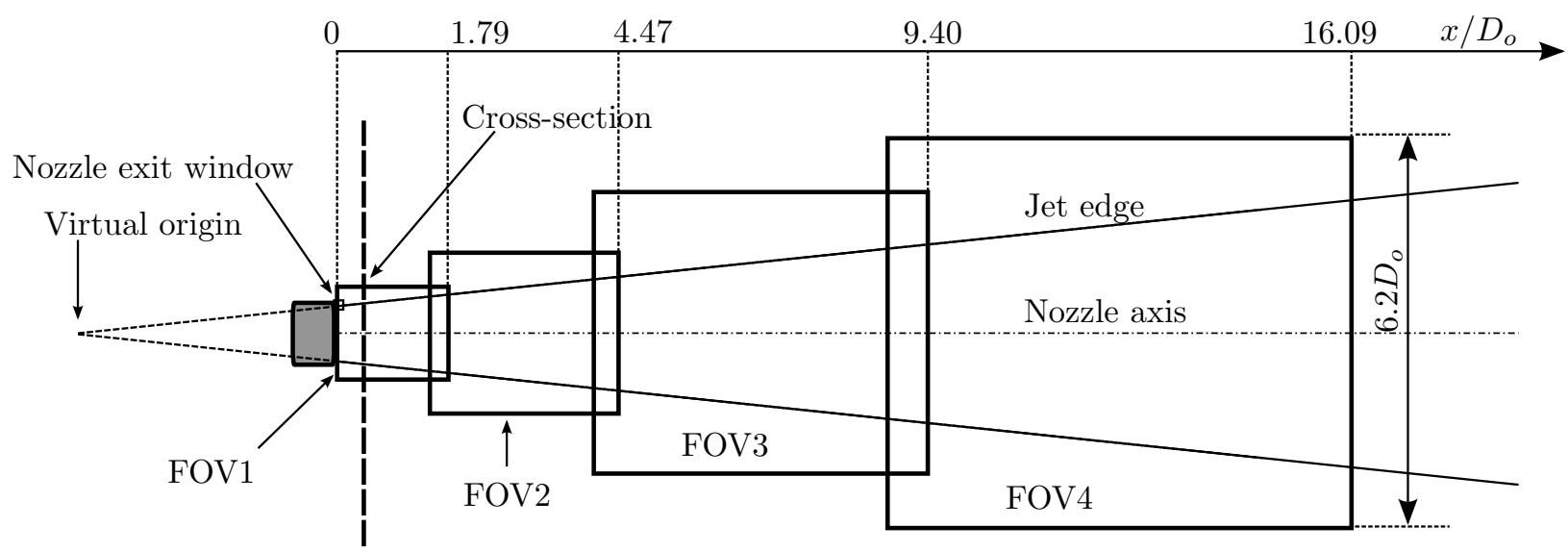

FIG. 5: The positions of the four overlapping rectangular PIV measurement windows (fields of view: FOV1-FOV4) and the nozzle exit PIV window; see Table II for locations and dimensions. The time-averaged jet perimeter and virtual origin are superimposed to indicate where the jet fell relative to each window. The location of the cross-section perpendicular to the longitudinal nozzle axis at $x / D_{o}=0.5$ is marked by the vertical dashed line.

TABLE II: PIV measurement domains. Entries give the coordinates for the fields of view (FOVs) shown in Fig. 5. NE refers to the field of view immediately adjacent to the nozzle exit. The final two columns give the ratio of the length scale for the flow $b$ to the spatial resolution of the calculated vectors $R_{s}$, and ratio of the timescale for the flow $\tau$ to the temporal resolution $R_{t}$.

\begin{tabular}{llllllllllll}
\hline \hline FOV & $\begin{array}{l}x_{\min } \\
(\mathrm{mm})\end{array}$ & $\begin{array}{l}x_{\max } \\
(\mathrm{mm})\end{array}$ & $\begin{array}{l}y_{\min } \\
(\mathrm{mm})\end{array}$ & $\begin{array}{l}y_{\max } \\
(\mathrm{mm})\end{array}$ & $\frac{x_{\min }}{D_{o}}$ & $\frac{x_{\max }}{D_{o}}$ & $\frac{y_{\min }}{D_{o}}$ & $\frac{y_{\max }}{D_{o}}$ & $\begin{array}{l}R_{s} \\
(\mathrm{~mm})\end{array}$ & $b / R_{s}$ & $\tau / R_{t}$ \\
\hline $\mathrm{NE}$ & 0.3 & 20.4 & 41.6 & 58.4 & 0.00 & 0.20 & 0.41 & 0.57 & 0.1 & 8 & 0.006 \\
1 & 2.5 & 181.5 & -76.3 & 74.9 & 0.02 & 1.79 & -0.75 & 0.74 & 1.1 & $2-50$ & $0.006-0.6$ \\
2 & 148.1 & 454.5 & -130.5 & 128.0 & 1.46 & 4.47 & -1.28 & 1.26 & 1.9 & $30-35$ & $0.6-1.1$ \\
3 & 414.6 & 955.1 & -229.7 & 226.3 & 4.08 & 9.40 & -2.26 & 2.23 & 3.4 & $20-30$ & $1.1-1.4$ \\
4 & 885.7 & 1635.2 & -321.5 & 310.9 & 8.71 & 16.09 & -3.16 & 3.06 & 4.7 & $20-40$ & $1.4-1.5$ \\
\hline \hline
\end{tabular}

\section{Diagnostics}

The sequential image pairs, obtained using the experimental set-up described, were processed using a multi-pass cross-correlation algorithm to obtain the velocity vectors; see Appendix $B$ for more information.

A total of $1250 \mathrm{PIV}$ datasets acquired at $15 \mathrm{~Hz}$ in each FOV, i.e. over a period of $84 \mathrm{~s}$, were found to be sufficient for obtaining meaningful estimates of time-averaged quantities (specifically the axial $\bar{u}$ and radial $\bar{v}$ time-averaged velocities) and turbulent statistics (the standard deviation of the axial $\sigma_{u}$ and radial $\sigma_{v}$ velocity fluctuations). The averaging period was confirmed to be sufficient by the smooth velocity and turbulent intensity profiles (Sec. III). A comparison of a length scale characteristic of the local turbulence $b$ with the spatial resolution $R_{s}$ of our data reveals that we are able to resolve in detail the velocity field. Following Ezzamel et al. [27], $b$ 
was taken to be the local jet width; this scale varied from a minimum of $b \approx l \approx 2 \mathrm{~mm}$ adjacent to the nozzle (Table I) to a maximum of $b \approx 180 \mathrm{~mm}$ downstream. Accordingly, the spatial resolution of the data (penultimate column of Table II) is at least one order of magnitude less than the characteristic turbulent length scale in each field of view. By contrast, a comparison of a characteristic flow timescale $\tau$ with the temporal resolution $R_{t}$ of our data reveals that the resolution is not sufficient to extract turbulence spectra. A representative timescale for the flow $\left(\tau=b / \sigma_{u c}\right)$ was estimated based on the local characteristic length and velocity scales, the latter taken to be the standard deviation of the horizontal centreline velocity $\left(\sigma_{u c}\right)$; the subscript ' $c$ ' denotes centreline. The velocity scale was $\sim 5 \mathrm{~m} \mathrm{~s}^{-1}$ adjacent to the source (NE, Table II) and $\sim 0.5 \mathrm{~m} \mathrm{~s}^{-1}$ at the furthest measured downstream location (in FOV4). As a consequence, turbulent timescales are approximately $\tau=1 \times 10^{-3} \mathrm{~s}$ adjacent to the source and $\tau=0.1 \mathrm{~s}$ at the furthest downstream measurement location. Therefore, at $15 \mathrm{~Hz}$ the temporal resolution of our data was not sufficient to extract spectral information (see the last column of Table II).

Although our spatial resolution is sufficient to describe the velocity field in detail, to ensure good quality results we took all reasonable steps to minimise measurement errors as outlined in Appendix C. Errors associated with the PIV algorithm were found to be below $2 \%$ of the total displacement identified at worst. Other sources of error, including those due to peak-locking effects and due to the relative placement of camera and laser were minimised before data acquisition and can be considered negligible.

For completeness, the first- and second- order velocity statistics used in Sec. III are defined below. The time-averaged axial velocity $\bar{u}$ and the standard deviation of the axial velocity fluctuations $\sigma_{u}$ are

$$
\begin{aligned}
\bar{u}(x, r, \theta) & =\frac{1}{n_{a v}} \sum_{n=1}^{n_{a v}} u(x, r, \theta, n), \\
\sigma_{u}^{2}(x, r, \theta) & =\frac{1}{n_{a v}} \sum_{n=1}^{n_{a v}}(u(x, r, \theta, n)-\bar{u}(x, r, \theta))^{2},
\end{aligned}
$$

where $u$ is the instantaneous axial velocity, $n$ the dataset number and $n_{a v}=1250$ the total number of datasets that were averaged; the time-averaged radial velocity $\bar{v}$ and associated standard deviation $\sigma_{v}$ are calculated similarly. The time-averaged circumferential velocity is

$$
\bar{u}_{\theta}(x, r, \theta)=\frac{1}{n_{a v}} \sum_{n=1}^{n_{a v}} u_{\theta}(x, r, \theta, n)
$$

where $u_{\theta}$ denotes the instantaneous circumferential velocity. Denoting the radius of the flow induced through the core as $r_{i}(x)$ (Fig. 6), a sectional average for the mean streamwise velocity within the bounded induced-flow region $\bar{u}_{i}(x)$ and the associated standard deviation $\sigma_{i}(x)$ were evaluated as

$$
\begin{aligned}
\bar{u}_{i}(x) & =\frac{1}{\pi r_{i}^{2}} \int_{0}^{2 \pi} \int_{0}^{r_{i}} \bar{u}(x, r, \theta) r \mathrm{~d} r \mathrm{~d} \theta \\
\sigma_{i}^{2}(x) & =\frac{1}{\pi r_{i}^{2}} \int_{0}^{2 \pi} \int_{0}^{r_{i}}\left(\bar{u}(x, r, \theta)-\bar{u}_{i}(x)\right)^{2} r \mathrm{~d} r \mathrm{~d} \theta .
\end{aligned}
$$

Note that at the source $r_{i}(0)=D_{i} / 2$; elsewhere, we define $r_{i}$ by $\sigma_{u}\left(x, r_{i}, \theta\right) / \bar{u}_{\max }(x)=0.05$, with $\bar{u}_{\max }(x)$ denoting the maximum streamwise velocity on the $r-\theta$ plane. Finally, the mean axial velocity $\bar{u}_{p}$ around the circumference of a circle with radius $r=$ const. and the standard deviation 


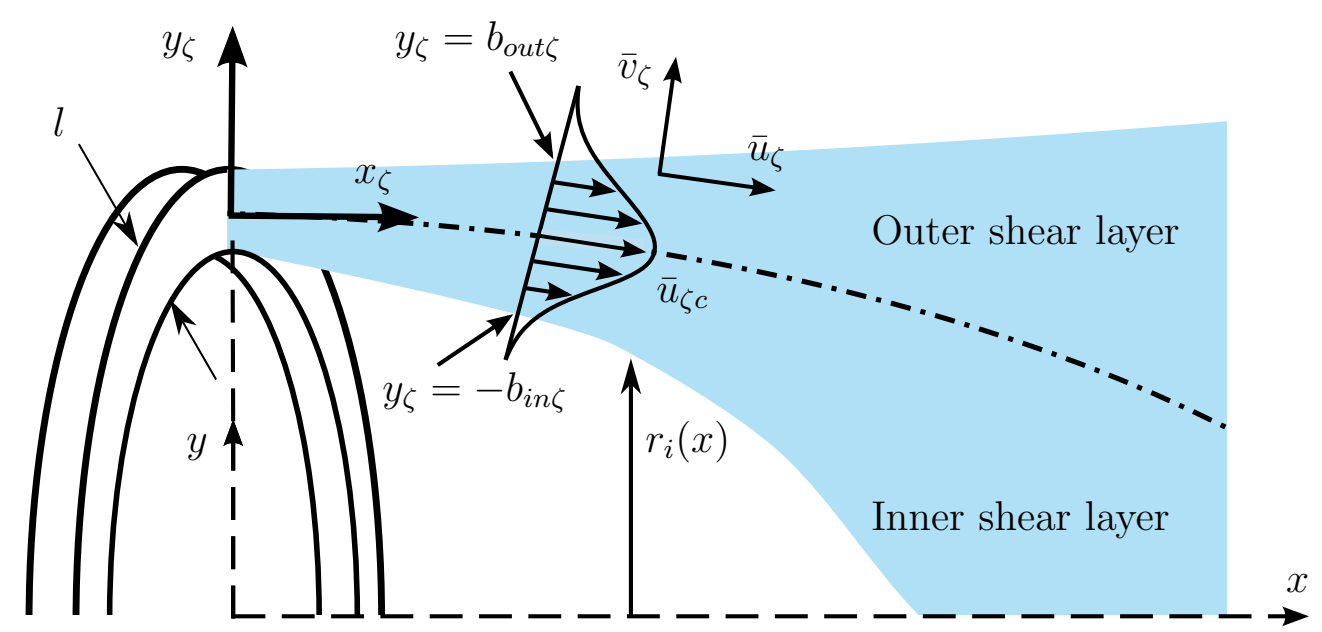

FIG. 6: Local coordinate system $\left(x_{\zeta}, y_{\zeta}\right)$ for the near-field region. The dot-dashed line indicates the 'centreline' along which the streamwise velocity takes a maximum value $\bar{u}_{\zeta}\left(x_{\zeta}, 0\right)=\bar{u}_{\zeta c}$. The coordinate system follows this high-velocity centreline from the slot such that $x_{\zeta}$ is tangential to, and $y_{\zeta}$ is perpendicular to, this centreline. The velocities $\bar{u}_{\zeta}$ and $\bar{v}_{\zeta}$ are those in the streamwise and cross-stream directions, respectively.

of this velocity $\sigma_{p}$ are

$$
\begin{aligned}
& \bar{u}_{p}(x)=\frac{1}{2 \pi} \int_{0}^{2 \pi} \bar{u}(x, r=\text { const. }, \theta) \mathrm{d} \theta \\
& \sigma_{p}^{2}(x)=\frac{1}{2 \pi} \int_{0}^{2 \pi}\left(\bar{u}(x, r=\text { const. }, \theta)-\bar{u}_{p}(x)\right)^{2} \mathrm{~d} \theta
\end{aligned}
$$

the subscript ' $p$ ' denoting that these quantities represent an average over the perimeter $[0,2 \pi]$.

\section{RESULTS \& DISCUSSION}

The flow visualisation shown in Fig. 2 reveals a number of distinguishing features that are characteristic of this previously unstudied class of jet. The image captures an instantaneous snapshot of the $x-y$ plane extending from the source to approximately $5 D_{o}$ downstream. In the near nozzle region, the fine jet issuing horizontally from the slender annular slot can be clearly seen, as can the open core of the nozzle. Immediately apparent is an inward curvature of this jet which subsequently coalesces to bound an internal region of induced flow. Entrainment into the inner shear layer of the jet is responsible for inducing this flow: a laminar flow that is drawn through the open core in the direction (left to right) of the jet. The resulting bullet-shaped internal induced-flow region is unique to these open-core ventilated jets. In contrast with observations of closed-core annular jets [e.g. 14, 15], a large-scale unsteady recirculation region immediately downstream of the nozzle is not observed. Pre-coalescence, the external envelope of the jet does not vary significantly in width, however, further downstream the jet expands and is similar in appearance to a classic round jet that develops from a circular orifice. These general features of the annular-jet flow are confirmed in the complementary contour plots of time-averaged axial velocity $\bar{u} / U_{0}$; Fig. 7 (a) depicts the entire measurement domain and Fig. 7(b) the near-field region of jet convergence and bounded region of 

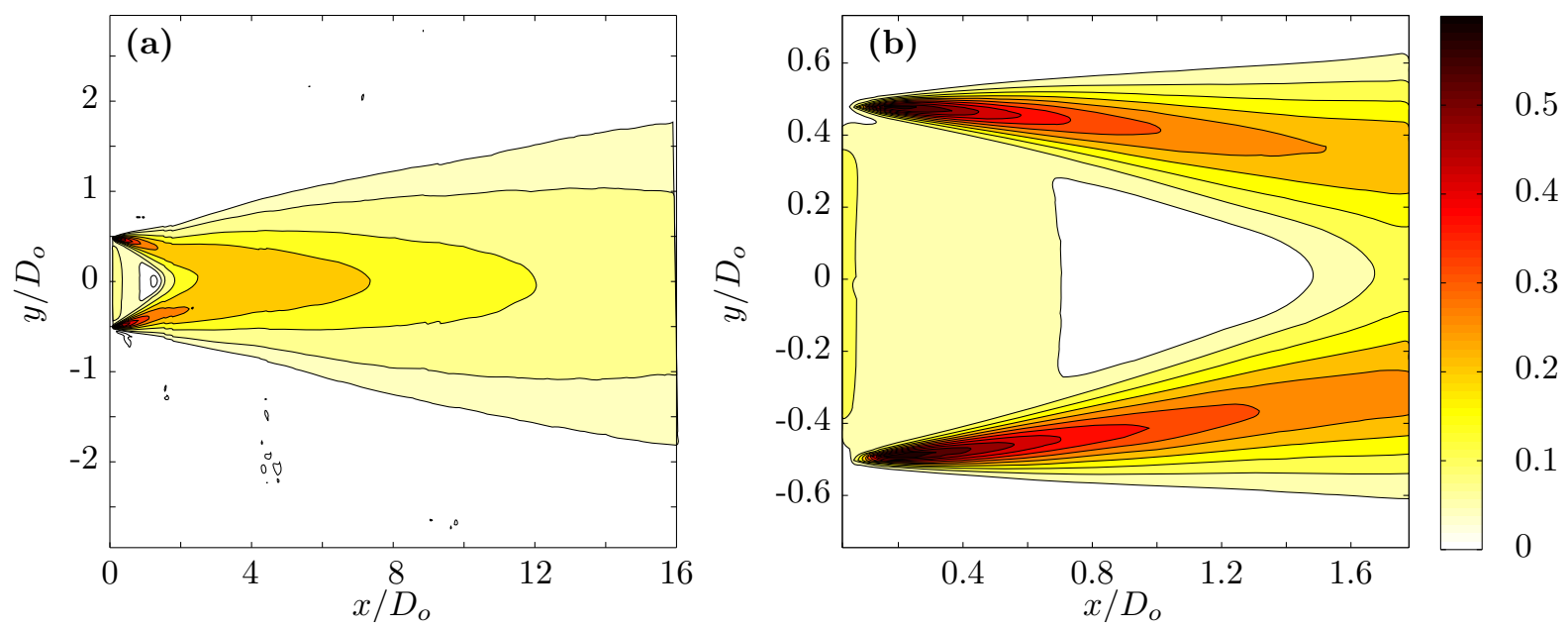

FIG. 7: Contour plots of the time-averaged axial velocity $\bar{u}(x, y)$ for the ventilated annular jet $\left(D_{i} / D_{o}=0.968, R e_{l} \approx 3200\right)$ in the $x-y$ plane. (a) The entire measurement domain using plotted data from FOV1-FOV4. (b) Region adjacent to the nozzle corresponding to FOV1. The colour bar indicates the non-dimensional streamwise velocity $\bar{u} / U_{0}$. Close to the slot $\left(x \lesssim 0.1 D_{o}\right)$

the velocities in the jet are not adequately captured by the PIV measurements due to the relatively thin jet $(O(l=1.65 \mathrm{~mm}))$ when compared to the resolution of FOV1 $\left(R_{s}=1.1 \mathrm{~mm}\right.$, Table II).

induced flow. The variation of velocity within the jet and ambient is examined in the subsections that follow.

Regarding terminology, we refer to the high-velocity core of the curved merging jet and of the merged jet as a 'centreline'. With respect to the near field, development of the flow in the direction of the centreline is referred to as 'streamwise' and sections perpendicular to the centreline as 'cross stream'. Figure 6 shows such a high-velocity centreline as a dot-dashed line and introduces the local coordinate system for the near field $\left(x_{\zeta}, y_{\zeta}\right)$ that follows the centreline; the subscript $\zeta$ signifies values in this coordinate system so that, for example, $u_{\zeta c}$ denotes the centreline streamwise velocity. With respect to the far field, where the time-averaged position of the high-velocity centreline is coincident with the longitudinal axis of the nozzle, flow development in the direction of this axis is referred to as 'axial' and sections perpendicular to this axis as 'radial', so as to distinguish from the near-field descriptions.

To identify the location of the reattachment point, i.e. where the high-velocity centreline merges (Fig. 1), in Fig. 8 we plot the axial velocity $\bar{u}(x, y=0)$ between $0<x / D_{o} \lesssim 9.5$. Note that the axial velocity increases for $1.5 \lesssim x / D_{o} \lesssim 4.5$ and thereafter decreases. According to Warda et al. [20], the increase in velocity corresponds with the coalescing of the jet, however, once the jet has completely coalesced, i.e. beyond the reattachment point, the velocity along the nozzle axis decays. This locates the reattachment point at $x_{r e} \approx 4.5 D_{o}$.

The results of Ko and Chan [15] suggest that an unventilated annular jet (with an identical diameter ratio to that examined herein) reattaches approximately $1.1 D_{o}$ downstream of the nozzle. The reattachment of the ventilated annular jet further downstream is readily attributed to the open core of the annulus; fluid is induced through the core, reducing the pressure difference between the central region and the ambient, resulting in a diminished jet curvature and consequently reattachment further downstream.

The observations above, and results that follow, show that the ventilated annular jet can be split 


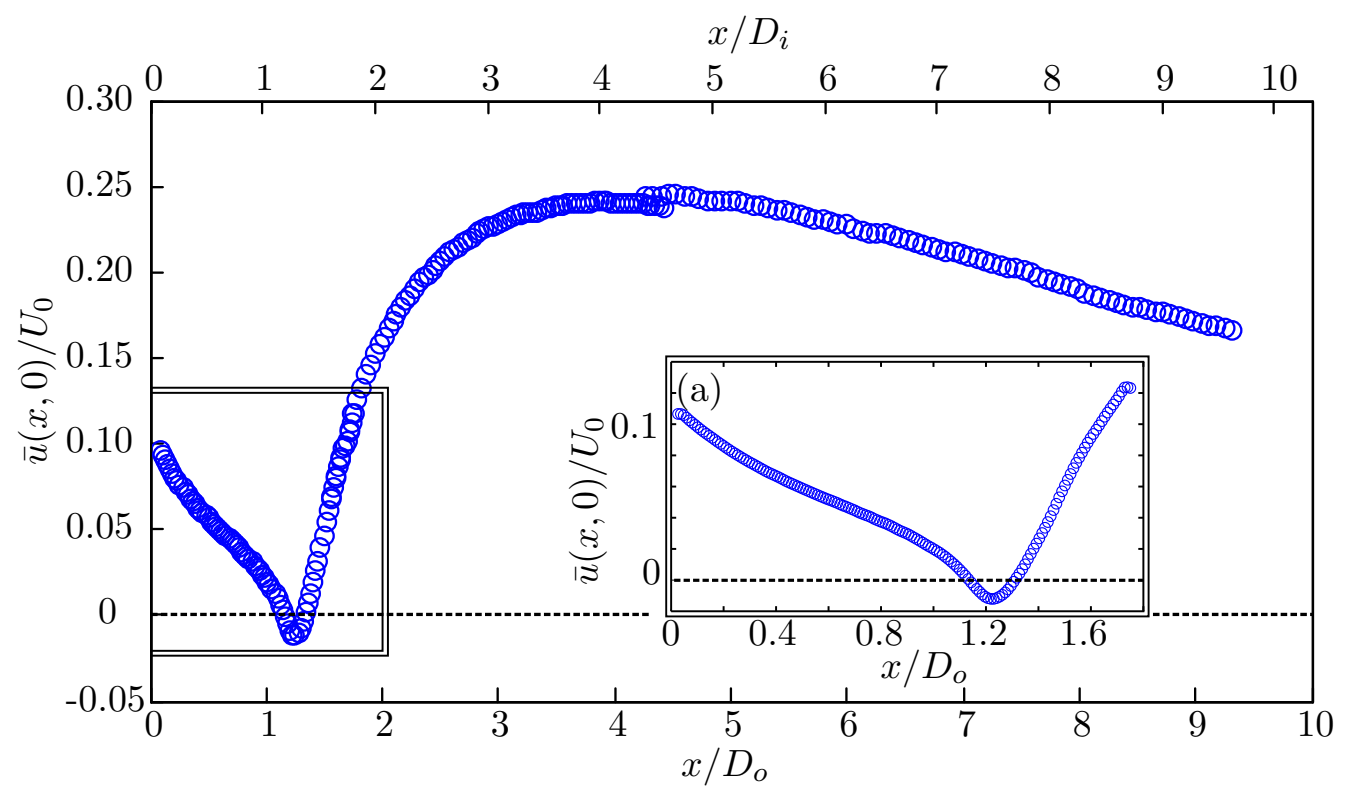

FIG. 8: Dimensionless axial velocity along the longitudinal nozzle axis $\bar{u}(x, 0) / U_{0}$ for $0<x / D_{o} \lesssim 9.5$. The reattachment point is located at $x / D_{o} \approx 4.5$ where the velocity is at a maximum. The internal bounded region of induced flow extends for $0 \leq x / D_{o} \lesssim 1.2$. Insert (a) shows a zoomed view for $0 \leq x / D_{o} \lesssim 1.8$. Note the region of flow reversal (negative velocity) for $1.11 \lesssim x / D_{o} \lesssim 1.30$

into four regions, as indicated on Fig. 2, each with a distinct behaviour: a bounded induced-flow region (Sec. III B), a near-field region of coalescence (Sec. III C, Sec. III D), a transitional region (Sec. IIIF) and a far-field round-jet-like region (Sec. III E). The geometry of an annular nozzle offers multiple lengths on which one could choose to scale the data. When considering the nearfield development of the flow (Sec. III C, Sec. IIID) we scale on the slot width $l$. The streamwise development in the far field (Sec. IIIE 2) is scaled on the round-jet length scale $\sqrt{A_{0}}$. In the remaining regions we scale on the outer diameter $D_{o}$ as is standard in the unventilated annular jet literature. While we note that for the bounded induced-flow region (Sec. III B), $D_{i}$ characterises the distance separating the opposite sides of the inner shear layer at the source, for our slender source $D_{i} \approx D_{o}$ and therefore we scale this region on $D_{o}$ for consistency. Before examining the flow within the four regions, we assess the fidelity of our measurements by evaluating the momentum integral $M(x)$.

\section{A. Momentum integral}

For an isothermal free jet, the momentum integral is independent of the distance downstream of the source [28]. To the second order, the momentum integral

$$
M(x)=\underbrace{\pi \int_{-\infty}^{\infty} \bar{u}^{2} r \mathrm{~d} r}_{M_{m}(x)}+\underbrace{\pi \int_{-\infty}^{\infty}\left(\overline{u^{\prime 2}}-\overline{v^{\prime 2}}\right) r \mathrm{~d} r}_{M_{t p}(x)}
$$

[cf. 29] was evaluated based on our planar PIV measurements of $\bar{u}, u^{\prime}$ and $v^{\prime}$. In (10), $u^{\prime}$ and $v^{\prime}$ denote the axial and radial fluctuating velocity components, respectively. 


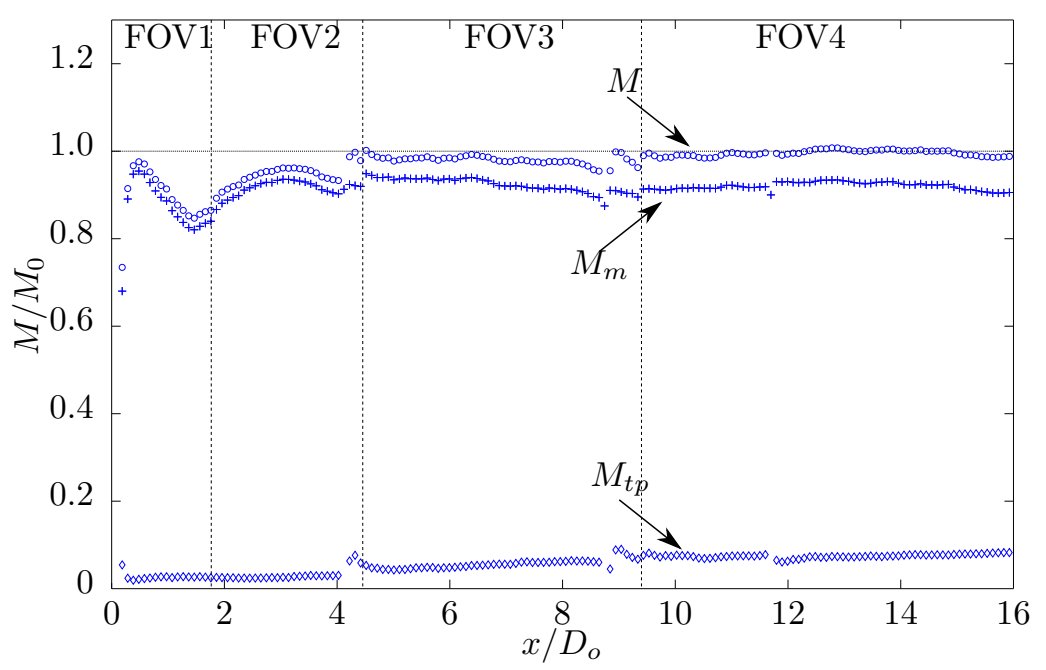

FIG. 9: Variation of the dimensionless momentum integral $M / M_{0}$ with distance downstream, from (10), for the ventilated annular jet. Also shown are the contributions from the mean flow, $M_{m} / M_{0}$, and from the turbulent fluctuations and axial pressure integral, $M_{t p} / M_{0}$. The source momentum flux was estimated as $M_{0}=Q_{0}^{2} / A_{0}$. The vertical dotted lines indicate the downstream extents of the fields of view (cf. Table II).

The resulting estimates of $M(x)$, scaled on the source momentum flux $M_{0}$, are plotted in Fig. 9; also shown are the variation of the mean flow component $M_{m}(x)$, and the contribution $M_{t p}(x)$ attributed to the turbulent quantities and axial pressure integral.

For $x \gtrsim 4 D_{o}, M / M_{0} \approx 1$ indicating that the momentum integral of the jet is indeed conserved. Moreover, there is no sign of a decay in the momentum integral for the measurements made furthest downstream, indicating that the test chamber was sufficiently large, and specifically that the jet was not unduly influenced by a return flow in the chamber. Evidently, on moving downstream the turbulent contribution $M_{t p}$ increases and attains approximately $5-7 \%$ of the source momentum flux. This downstream increase is attributed to the increase in the turbulent intensities (see Fig. 17 in Sec. III E) as the jet evolves towards a state of approximate self-similarity.

For $x \lesssim 4 D_{o}$, the normalised momentum integral estimates fall significantly below unity. Drawing firm conclusions as to the reasons for the trend observed is not straightforward due to the near-source flow asymmetries, the dynamics associated with jet coalescence (the reattachment point falling at $x \approx 4.5 D_{o}$ ) and to the chosen fields of view (which overlap at $x \approx 4 D_{o}$ ). Whilst $M(x) / M_{0}<1$ is not expected on physical grounds as no sinks of momentum were present, momentum integral estimates calculated from measurements made on the single $x-y$ plane are not representative of the true momentum integral. Although it is unclear as to what degree the jet is symmetrical before $x / D_{o}=4$, these findings suggest the jet is symmetrical for $x / D_{o} \gtrsim 4$.

\section{B. Induced flow}

The pattern of streamlines characteristic of the near-field flow is shown in Fig. 10. The region shown extends from the nozzle exit into the coalescence region and shows the flow within and induced by the annular jet. The streamlines were calculated using MATLAB's built in streamline function, hence the distance separating neighbouring streamlines does not correspond to a constant change in the stream function. The stream function is not used for displaying the streamlines as it is not trivial to accurately calculate the stream function from the PIV vectors as any noise or 


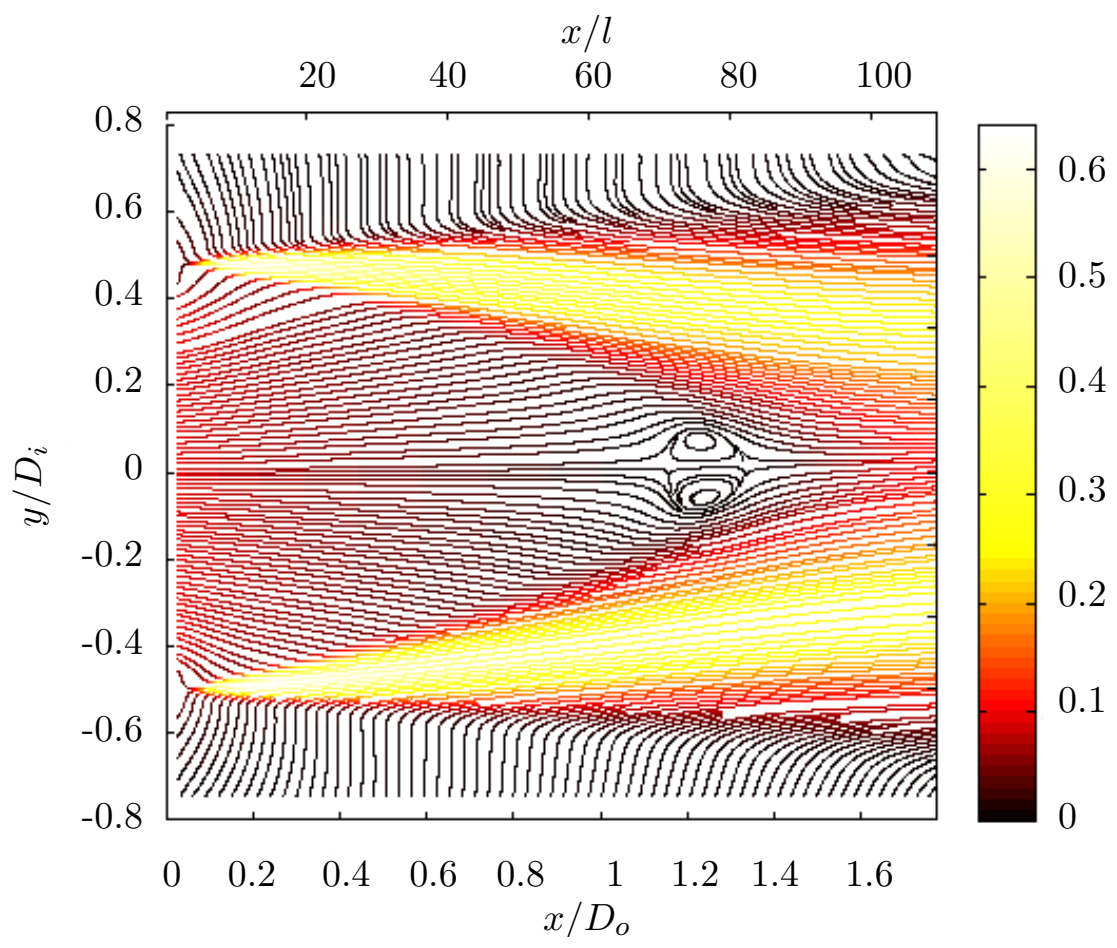

FIG. 10: Time-averaged streamline pattern shown on a section through the ventilated annular jet that extends along the longitudinal axis from the nozzle exit to the jet-coalescence region. Colour indicates the non-dimensional velocity magnitude $\sqrt{\bar{u}^{2}+\bar{v}^{2}} / U_{0}$ along streamlines.

errors present within the PIV data will tend to propagate on integration. The streamline pattern reveals that the entrainment of ambient fluid into the outer shear layer of the jet (external jet perimeters at top and bottom of image) induces a flow in a direction perpendicular (approx.) to the local high-velocity centreline. By contrast, entrainment into the inner shear layer induces a flow perpendicular to this, drawing fluid through the open core in the direction of the nozzle axis.

\section{(i) Inner shear layer}

One of the distinguishing features of the streamline pattern for the internal induced flow is a relatively small-scale region of recirculation situated along the axis of symmetry for $1.1 \lesssim x / D_{o} \lesssim$ 1.3. Examination of the flow visualisation image (Fig. 2) shows that the converging jet does not coalesce at a point, rather the downstream limit of the induced-flow region appears 'blunt' ended. Observations of the flow here showed complex self-interactions within the inner shear layer, with eddies crossing the longitudinal nozzle axis - in other words, a region where the jet self-entrained. As such, we believe the recirculation region seen in Fig. 10 to be a consequence of shear-layer interactions. Figure 8(a), which plots the normalised velocity $\bar{u}(x, 0) / U_{0}$ along the nozzle axis, reveals that the recirculating region comprises a region of reverse flow $\left(\bar{u}(x, 0) / U_{0}<0\right)$ bounded by two stagnation points, one at $x / D_{o} \approx 1.11$ and the other at $x / D_{o} \approx 1.30$. Moreover, the induced flow decelerates from a maximum velocity at the exit plane of the nozzle to a weakly negative velocity in the recirculation region and subsequently accelerates into the region of jet coalescence. This time-averaged picture implies that pressure is lowest at the plane of the open core, increases to a local maxima at the stagnation points, before decreasing again as the jet coalesces. To elaborate, the upstream stagnation point is expected to be at atmospheric pressure given that the upstream 
flow (induced through the core of the annulus) is inviscid. Due to the highly turbulent motions within the recirculating vortex, the downstream stagnation point is expected to be at a pressure below atmospheric.

Defining the streamwise extent $\left(x_{\text {merge }}\right)$ of the bounded induced-flow region to be the point at which the inner shear layer first self-interacts based on the time-averaged picture of the flow, i.e. as the upstream end of the recirculation region, gives $x_{\text {merge }} / D_{o} \approx 1.11$. Alternatively, on specifying the perimeter of the inner shear layer as the locus of points along which the streamwise velocity has fallen to $1 / e$ (where $\ln (e)=1$ ) of the peak high-velocity centreline value, a second estimate of $x_{\text {merge }} / D_{o}=1.60$ can be obtained from the intersection of the loci.

The flow induced through the open core of the annulus is subsequently entrained into the jet and thereby serves to enhance the volume flux in the near field when compared with the closed-core case. On calculating the mean velocity of the flow induced through the $r-\theta$ plane at $x / D_{o}=0.5$ (Fig. 21), using (6) and (7) with an estimate of $r_{i} / D_{o}=0.35$, yields $\bar{u}_{i} / U_{0}=0.056$ with a standard deviation of $\pm 2 \%\left(\sigma_{i} / U_{0}=0.001\right)$. Therefore, although the jet has a weak $\theta$-dependence in the near field (Appendix D), it appears that the induced flow has no such $\theta$-dependence. We may then reliably estimate the volume flux induced through the core of the annulus by evaluating $Q_{a}=\int_{0}^{2 \pi} \int_{0}^{D_{i} / 2} \bar{u}(x=0) r \mathrm{~d} r \mathrm{~d} \theta=\pi \int_{-D_{i} / 2}^{D_{i} / 2} \bar{u}(x=0) y \mathrm{~d} y$. Based on this we obtain $Q_{a} / Q_{0}=1.2$.

\section{(ii) Outer shear layer for $x \leq x_{\text {merge }}$}

Having now estimated the total volume flux entrained by the inner shear layer as $Q_{a}=1.2 Q_{0}$, it is of interest to compare this with the total volume flux $Q_{e}$ that is entrained by the outer shear layer over a comparable streamwise distance, namely for $0 \leq x \leq x_{\text {merge }}$. To estimate $Q_{e}$ we first note that the volume flux of the flow at $x=x_{\text {merge }}, Q\left(x_{\text {merge }}\right)$, consists of contributions from the source $Q_{0}$, from fluid induced through the open core $Q_{a}$ and fluid entrained into the outer shear layer $Q_{e}$. It follows that

$$
\frac{Q_{e}}{Q_{0}}=\frac{Q\left(x_{\text {merge }}\right)}{Q_{0}}-\frac{Q_{a}}{Q_{0}}-1,
$$

where the volume flux of the jet $Q$ is calculated within a region bounded by a perimeter located at $y= \pm y_{w}$, defined such that $y_{w}>\left|D_{o} / 2\right|$ and $\bar{u}\left(x, y_{w}\right) / \bar{u}_{\max }=0.05$. This definition of $y_{w}$, obtained through a process of trial and error, bounds the external jet perimeter such that estimates of volume flux remain continuous at the boundary of two measurement windows. Thus,

$$
Q(x)=\pi \int_{-y_{w}}^{y_{w}} \bar{u}(x, y) y \mathrm{~d} y .
$$

As this definition encompasses all the fluid contained between the limits of integration, the internal induced flow is included in the resulting estimate of $Q(x)$. Evaluating (12) at $x=x_{\text {merge }}$, by which point the entirety of the internal induced flow has been entrained by the inner shear layer, allows us to estimate the volume flux entrained by the outer shear layer between $0 \leq x \leq x_{\text {merge }}$. Taking $x_{\text {merge }}=1.11 D_{o}$ we obtain $Q_{e} / Q_{0}=1.5$. Alternatively, on taking $x_{\text {merge }}=1.60 D_{o}$ we obtain $Q_{e} / Q_{0}=2.0$. Both estimates are approximate due to the asymmetries in the jet near the nozzle (see Appendix D).

Evidently, both the inner and outer shear layers entrain different amounts of fluid. This difference in entrainment can be explained by the differing surface areas of the interfaces over which this fluid is entrained - the area of the inner entrainment surface being the smaller (Fig. 2). On characterising the perimeter of both shear layers by the $1 / e$ velocity contours (and therefore with $\left.x_{\text {merge }}=1.60 D_{o}\right)$, we obtain a surface of area $I S L=4.2 \pi\left(D_{o} / 2\right)^{2}$ for the inner shear layer 
and of $O S L=7.1 \pi\left(D_{o} / 2\right)^{2}$ for the outer shear layer. The ratio of surface areas for these shear layers is approximately equal to the ratio of volume fluxes entrained (at $x_{\text {merge }}=1.60 D_{o}$ ), i.e. $Q_{e} / Q_{a}=1.66 \approx 1.69=O S L / I S L$, where the small difference can be attributed to experimental uncertainty.

\section{Jet development adjacent to the nozzle exit}

We proceed by examining the development of the jet immediately downstream of the slot, i.e. upstream of the region we refer to as 'near field' in Fig. 2. We recall the slot has dimension $l$ and planar PIV measurements were recorded between $x / l=0.2$ and $x / l=12$ (Table II, i.e. for $0.3 \leq x \leq 20.4 \mathrm{~mm}$ ). At first sight, the cross-stream variation in $\bar{u}_{\zeta}$ recorded immediately downstream of the slot (at $x_{\zeta} / l=0.2$ ), Fig. 11(a), appears to indicate that the velocity profile at the source could be reasonably approximated by a 'top-hat'. Closer examination reveals that the profile more closely resembles the 'saddle-back' velocity profile that occurs in jets issuing from a sharp-edged orifice [30]. The standard deviation $\sigma_{\zeta u}$ of the streamwise velocity (Fig. 11a dotted line) shows that the turbulence intensities peak within the shear layers and are approximately $5 \%$ $\left(\sigma_{\zeta u} / \bar{u}_{\zeta} \approx 0.04 / 0.85\right)$ within the core of the jet.

The colour map of axial velocity $\bar{u}$, Fig. 11(b), indicates that the trajectory of the jet in this region remains close to horizontal. The coordinate $y$ has been translated in the plot so that the longitudinal axis $\left(\left(y-\left(D_{i}+D_{o}\right) / 4\right) / l=0\right)$ bisects the slot. Moreover, measurements of the time-averaged streamwise velocity along the high-velocity centreline $\bar{u}_{\zeta c} / U_{0}$, Fig. $11(\mathrm{c})$, indicate that the velocity within the core remains almost unchanged for a distance of approximately $4-5 l$ downstream; a potential core of a length between 4-6l is in good agreement with the planar-jet literature [e.g. 31, 32]. It is also evident in Fig. 11(c) that the dimensionless velocity of the fluid exiting the slot in the $x-y$ plane is not equal to unity; this is attributed to asymmetries in the flow issuing from the nozzle (Appendix D).

\section{The near-field jet}

We now examine the flow further from the slot $\left(x / l>12\right.$ or, equivalently, $\left.x / D_{o}>0.2\right)$, where the curvature of the jet becomes significant, and into the region in which the shear layer coalesces. Our primary focus is on the streamwise development of velocity and turbulent intensity (Secs. III D 1-III D 2).

Moving away from the source the annular jet collapses towards the longitudinal axis of the nozzle and we attributed this to the pressure difference between the ambient and the internal region of induced flow (Sec. III B). For this 'near-field' region we introduced a coordinate system $\left(x_{\zeta}, y_{\zeta}\right)$ which follows the high-velocity jet centreline (Fig. 6), such that $y_{\zeta}>0$ represents locations within the outer shear layer and the ambient, and $y_{\zeta}<0$ represents locations within the inner shear layer and the internal induced-flow region. Within the near-field region, the high-velocity centreline merges at the reattachment point and is therefore not coincident with a streamline, i.e. we anticipate some cross-centreline flow. For sufficiently small $x_{\zeta} / l$ the jet appears to be horizontal in the flow visualisation (see also Fig. 11b) and thereafter its curvature increases. To follow the curving high-velocity centreline, a curve defined by a first-order Fourier series of the form $a_{0}+a_{1} \cos (p x)+a_{2} \sin (p x)$, for constants $a_{i}(i=0,1,2)$ and $p$, was fitted to the data points with the largest velocity magnitude. Profiles were found by performing cubic interpolation perpendicular to this curve.

For the profiles that follow in Sec. III D 1 (Figs. 12, 13), velocity is scaled on $\bar{u}_{\zeta c}$, the crossstream coordinate $y_{\zeta}$ is scaled on the width $b_{\zeta}$ of the outer shear layer (where $b_{\zeta}$ is defined such 

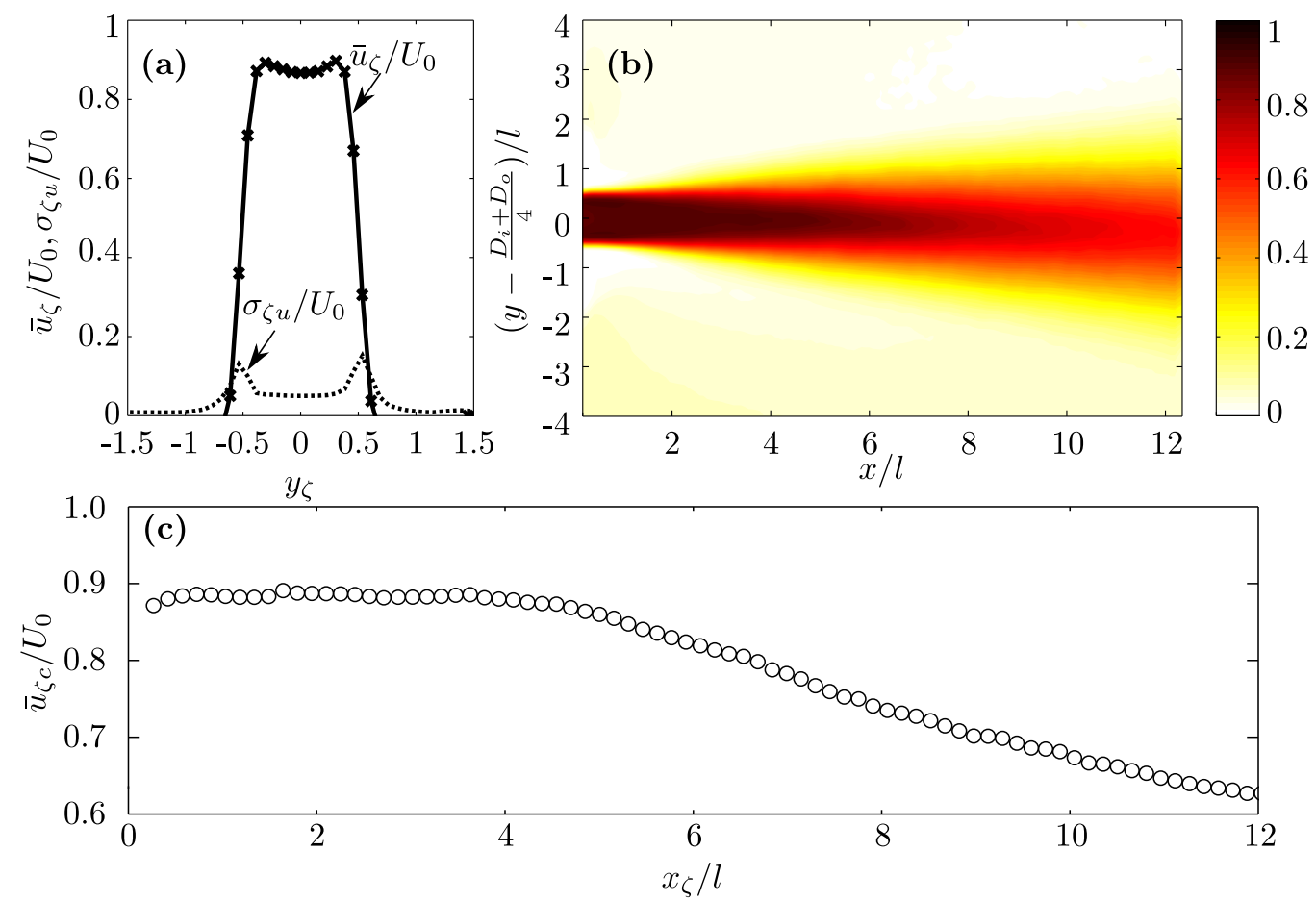

FIG. 11: Near-slot region. (a) Time-averaged cross-stream profile of streamwise velocity recorded immediately downstream of the exit plane at $x_{\zeta} / l=0.2$; measurement locations are marked with

a cross $\times$. (b) Colour map of time-averaged axial velocity downstream of the exit plane; the colour bar shows $\bar{u} / U_{0}$. (c) Time-averaged dimensionless streamwise velocity $\bar{u}_{\zeta c} / U_{0}$ along the high-velocity centreline exiting the slot; $x_{\zeta}$ denotes the distance along this centreline, Fig. 6.

that $\left.\bar{u}_{\zeta}\left(x_{\zeta}, b_{\zeta}\right)=\bar{u}_{\zeta c}\left(x_{\zeta}\right) / e\right)$, and we continue to scale distances in the streamwise direction on the slot width $l$. Note that on profiles for which $x_{\zeta} / l \geq 85$, the data point marked at the largest negative value of $y_{\zeta} / b_{\zeta}$ lies on the symmetry axis of the nozzle (i.e. on $y=0$ ).

\section{Velocity $\&$ intensity profiles}

From the profiles of time-averaged streamwise velocity $\bar{u}_{\zeta}\left(x_{\zeta}=\right.$ const., $\left.y_{\zeta}\right)$ plotted in Fig. 12(a), it is evident the structure of the outer shear layer $\left(y_{\zeta}>0\right)$ remains relatively unchanged over the measurement domain considered $\left(15 \leq x_{\zeta} / l \leq 265\right)$. The profiles appear to be approximately self-similar and follow closely the Gaussian $\bar{u}_{\zeta} / \bar{u}_{\zeta c}=\exp \left(-y_{\zeta}^{2} / b_{\zeta}^{2}\right)$. Whilst there is some evidence that a subset of the corresponding profiles for the inner shear layer show a partial collapse (see profiles for $x_{\zeta} / l=\{15+, 30 \bigcirc, 50 *\}$ ), there is clear evidence that, further downstream, the form of the profiles is influenced by the merging of the jet. Notably, the scaled velocities within the inner induced-flow region first decrease as the jet proceeds past the recirculation region (see $y_{\zeta} / b_{\zeta} \lesssim-2$ for $x_{\zeta} / l=\{70 \bullet, 85 \times\}$ and Sec. III B) before increasing as the inner shear layer combines. The streamwise velocity distribution in the outer shear layer is not noticeably affected by the merging of the jet.

We now turn our attention to the profiles of time-averaged cross-stream velocity $\bar{v}_{\zeta}\left(x_{\zeta}=\right.$ const., $\left.y_{\zeta}\right)$ plotted in Fig. 12(b). Merging has a significant effect on the cross-stream velocities as neither the structure of the inner nor the outer shear layer approach self-similar forms. The negative cross-stream velocity at the outer perimeter of the jet $\left(y_{\zeta} / b_{\zeta} \gtrsim 2\right)$ is a signature for 
TABLE III: Symbol with corresponding dimensionless streamwise distance downstream of the slot $x_{\zeta} / l$ used in the velocity and turbulent intensity profiles (Figs. 12, 13). Online: on travelling in the downstream direction, the colour of the symbols transmutes from blue to red.

\begin{tabular}{|c|c|c|c|c|c|c|c|c|c|c|c|c|}
\hline Symbol & + & 0 & $*$ & • & $x$ & $\square$ & $\diamond$ & $\triangleright$ & $\triangleleft$ & $\triangle$ & $\nabla$ & $\hat{z}$ \\
\hline$x_{\zeta} / l$ & 15 & 30 & 50 & 70 & 85 & 105 & 115 & 145 & 175 & 205 & 235 & 265 \\
\hline
\end{tabular}

the flow induced in the ambient. The positive cross-stream velocity at the inner perimeter of the jet $\left(y_{\zeta} / b_{\zeta} \lesssim-2\right)$ represents fluid that has been induced through the open core of the annulus. A notable feature apparent in Fig. 12(b) is the positive cross-stream velocity across the jet centreline for $x_{\zeta} / l \geq 30$, signifying a transport of fluid from the inner to the outer shear layer. While small compared to the streamwise velocity $\left(\bar{v}_{\zeta} / \bar{u}_{\zeta} \ll 1\right)$, this positive cross-stream velocity is not insignificant when compared to the maximum cross-stream velocity $\left(\bar{v}_{\zeta, \max }\right)$ in the jet with $\bar{v}_{\zeta}\left(x_{\zeta}, 0\right) / \bar{v}_{\zeta, \max }\left(x_{\zeta}\right) \approx 0.4-1.0$. Our data indicates that for $30 \leq x_{\zeta} / l \leq 85$, the cross-centreline velocity is almost constant, with a value of $\bar{v}_{\zeta}\left(x_{\zeta}, 0\right) / \bar{u}_{\zeta c}\left(x_{\zeta}\right) \approx 0.01$.

The non-zero velocity across the centreline suggests that the inner shear layer is being absorbed into the outer shear layer. Although, at a glance, one might be tempted to interpret this crosscentreline velocity as indicating that the inner shear layer entrains fluid at a greater rate than the outer shear layer, the analysis in Sec. IIIB indicates that the entrained volume flux is solely a function of the shear layers surface area.

Once the inner shear layer has merged, the magnitude of the velocity across the centreline $\bar{v}_{\zeta}\left(x_{\zeta}, 0\right)$ increases (note the profiles between $x_{\zeta} / l=\{115 \diamond\}$ and $x_{\zeta} / l=\{265 \bar{\zeta}\}$ ). Still further downstream, the high-velocity centreline merges (the reattachment point located at $x_{\zeta} / l \approx 277$ or, equivalently, $x / D_{o} \approx 4.5$, Sec. III). Thereafter, a single high-velocity centreline persists; after reattachment there is zero time-averaged volume flux across the centreline (see Sec. IIIE).

Profiles of streamwise $I_{\zeta u}\left(=\sigma_{\zeta u} / \bar{u}_{\zeta c}\right)$ and cross-stream $I_{\zeta v}\left(=\sigma_{\zeta v} / \bar{u}_{\zeta c}\right)$ turbulent intensity, where $\sigma_{\zeta u}$ and $\sigma_{\zeta v}$ denote the standard deviation of the respective velocity fluctuations, are plotted in Fig. 13. Although the furthest reaches of the outer shear layer $\left(y_{\zeta} / b_{\zeta} \gtrsim 1\right)$ are approximately self-similar for downstream distances exceeding $x_{\zeta} / l=30$, the furthest reaches of the inner shear layer $\left(y_{\zeta} / b_{\zeta} \lesssim-1.5\right)$ are self-similar only for the short span $30 \lesssim x_{\zeta} / l \lesssim 70$ and deviate from self-similarity as the inner shear layer begins to merge. Further downstream $\left(x_{\zeta} / l \gtrsim 115\right)$, the profiles closer to the axis $\left(y_{\zeta}=0\right)$ begin to evolve as the shear layer mixes and merges.

\section{Streamwise dependence}

Given the annular nozzle geometry considered, for which the nozzle radius is large compared with the width of the slot through which the jet issues $\left(D_{o} / 2 \gg l\right)$, and the existence of quasi selfsimilar streamwise velocity profiles (Sec. III D 1), one might reasonably anticipate a resemblance between the near-field jet from the annulus and the classic planar jet that issues from a slender rectangular slot. Indeed, as we shall see, planar-jet-like behaviour persists over a significant distance downstream of the exit slot. To enable direct comparisons with the classic results on planar jets we again scale lengths on the slot width $l$.

Figure 14(a) plots the variation of the normalised width of the inner shear layer, $b_{i n \zeta} / l$, and the outer shear layer, $b_{\text {out } \zeta} / l$, with distance along the high-velocity centreline $x_{\zeta} / l$. Data is plotted between the source and the location at which the high-velocity centreline merges (the reattachment point at $x_{\zeta} / l \approx 277$ ). The solid line shows the linear planar-jet scaling from Fischer et al. [25] with a gradient (growth rate) of 0.116 . Sufficiently close to the source, the growth rates of both the 

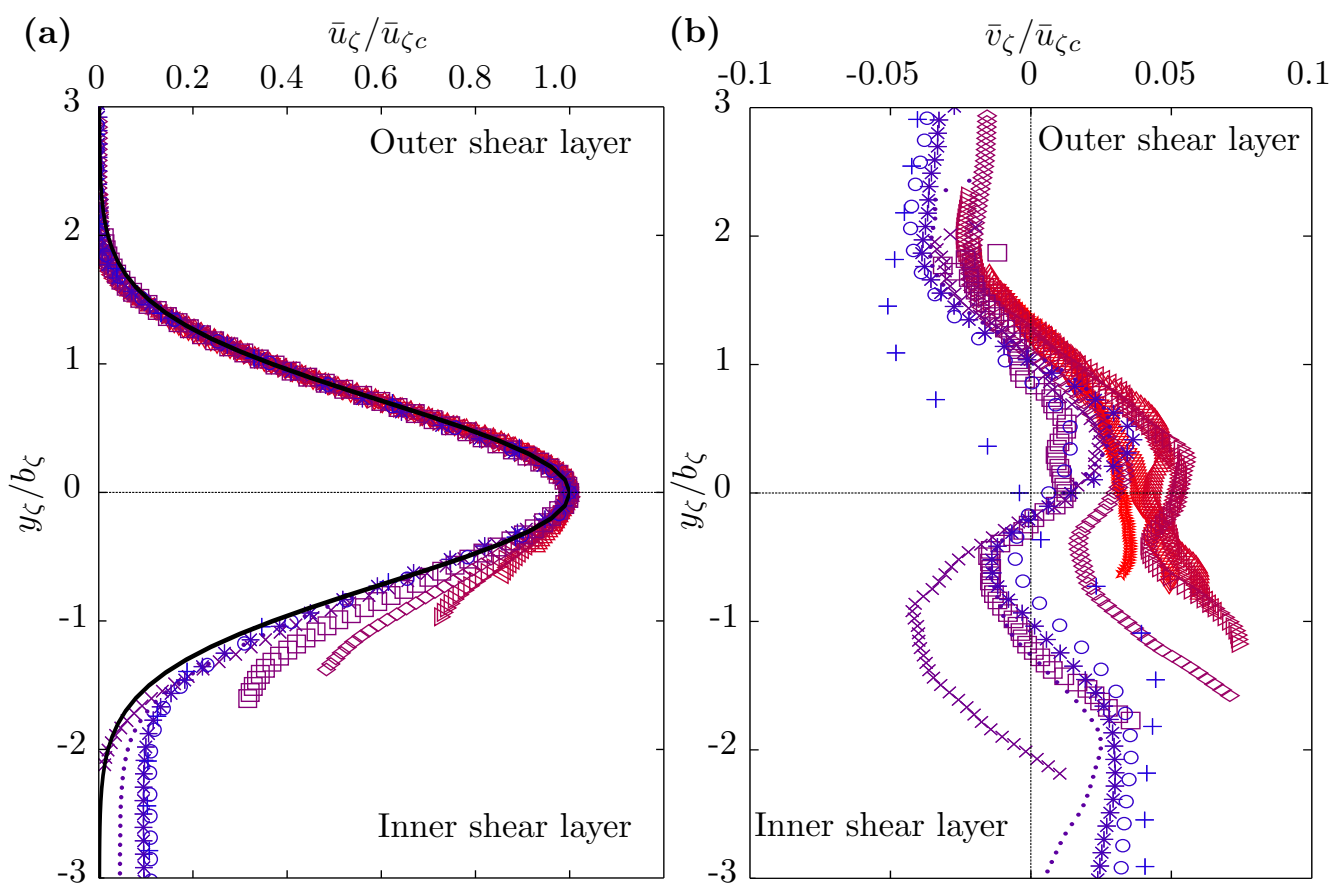

FIG. 12: Exit slot to reattachment region of the ventilated annular jet. Profiles of time-averaged: (a) streamwise velocity $\bar{u}_{\zeta}$ and (b) cross-stream velocity $\bar{v}_{\zeta}$ recorded at downstream distances from $x_{\zeta} / l=15(+)$ to $x_{\zeta} / l=265(\xi)$. Refer to Table III for downstream location corresponding to each symbol. The solid line in (a) is the Gaussian $\bar{u}_{\zeta} / \bar{u}_{\zeta c}=\exp \left(-y_{\zeta}^{2} / b_{\zeta}^{2}\right)$.

(a)

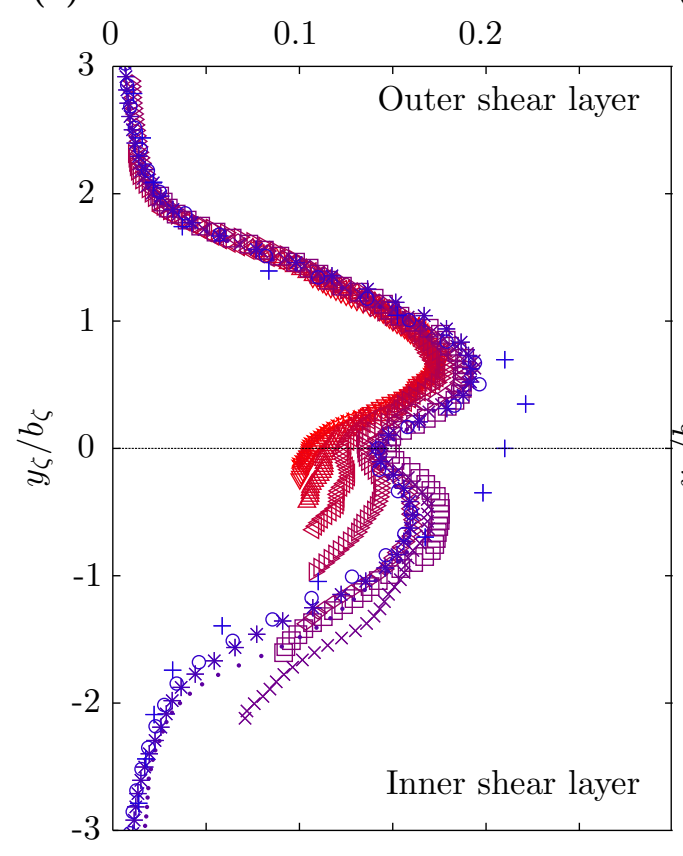

(b)

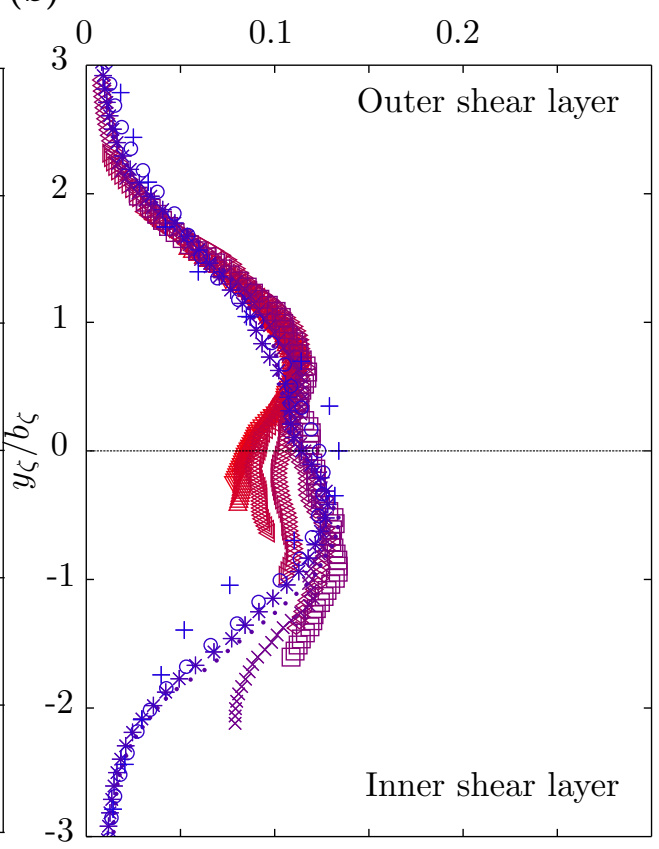

FIG. 13: Exit slot to reattachment region of the ventilated annular jet. Profiles of time-averaged: (a) streamwise turbulent intensity $I_{\zeta u}=\sigma_{\zeta u} / \bar{u}_{\zeta c}$ and (b) cross-stream turbulent intensity $I_{\zeta v}=\sigma_{\zeta v} / \bar{u}_{\zeta c}$. Refer to Table III for downstream location corresponding to each symbol. 
outer (o) and inner $(\square)$ shear layers are approximately linear and closely follow the growth rate of a planar jet.

For $x_{\zeta} / l \gtrsim 60$ the inner shear layer begins to rapidly widen, behaviour we attribute to selfinteractions. Further evidence of this local interaction is the presence of the recirculation region at $x_{\zeta} / l \approx 70$ (or, equivalently, at $x / l \approx 70$, see Fig. 10 and Sec. IIIB). The data ( $\square$ ) on Fig. 14 (a) is plotted until the inner shear layer begins to coalesce at $x_{\zeta} / l \approx 100$. This value yields the alternative estimate for the streamwise extent of the bounded induced-flow region discussed in Sec. III B, of $x_{\zeta \text { merge }} / l \approx 100$, or, equivalently, $x_{\text {merge }} / D_{o} \approx 1.60$.

For $x_{\zeta} / l \gtrsim 70$ the growth rate of the outer shear layer increases gradually, reaching a growth rate of 0.137 for $x_{\zeta} / l>150$ (dashed line on Fig. 14a). The increase in growth rate above that of the planar jet coincides with the merging of the annular jet. Still further downstream, the spreading rate begins to transition to that of a round jet; evidence of this behaviour is described in Sec. III E and Sec. IIIF.

Based on growth rates, we therefore assert that the annular jet closely resembles the planar jet for $0<x_{\zeta} / l \lesssim 60$. This assertion is further supported by the streamwise centreline velocity (Fig. 14b) which scales as $\bar{u}_{\zeta c} / U_{0} \propto\left(x_{\zeta} / l\right)^{-1 / 2}$ (solid line) for $x_{\zeta} / l<60$. For planar jets, it has long been established that $\bar{u}_{\zeta c} / U_{0}=$ const. $\left(x_{\zeta} / l\right)^{-1 / 2}[25]$. For our annular jet, the constant of proportionality is 2.5, whereas Fischer et al. [25] report a value of 2.41 for planar jets.

For $x_{\zeta} / l>100$ the rate of velocity decay in the jet from the annular slot is noticeably slower than for a planar jet. We attribute this to the transfer of momentum between the coalescing inner shear layer (annular case) rather than to transfers with the ambient (planar case). As we will see in Sec. III E, sufficiently far downstream (for $x_{\zeta} / l \gtrsim 540$ ) the streamwise velocity decays linearly, as is characteristic of a round jet [25]. In the range $60 \lesssim x_{\zeta} / l \lesssim 540$ our data suggests the annular jet may be regarded as in transition from planar to round-jet behaviour.

As a final note, the close agreement between classic scalings for a planar jet and our measurements provides us with a strong lead, namely the persistence of planar-jet-like behaviour indicates that the annular jet for $x_{\zeta} / l<60$ (or, equivalently, for $x / D_{o}<1$ ) is influenced primarily by the local geometry (via the length scale $l$ ) rather than by the annular geometry. However, the streamwise extent over which planar-jet behaviour holds is expected to be controlled by the dominant length scales of the nozzle $\left(D_{i} \& D_{o}\right)$, as these scales characterise the distance separating opposite sides of the (inner and outer) shear layers and of the jet centreline at the source.

\section{E. The far-field jet}

Turning our attention to the behaviour of the jet downstream of the region of coalescence, a natural question that arises concerns how the jet develops towards classic round-jet behaviour, as might reasonably be anticipated in the far field. Given this expectation, we revert to the global coordinate system $(x, y)$. We address this question by examining radial profiles of the firstand second- order velocity statistics, exploring the development towards a state of self-similarity. Our results (Appendix D) indicate that the flow shows no discernible swirl, may be regarded as axisymmetric at modest distances downstream and, as such, the $x-y$ plane interrogated, and on which the results of this section are based, is a representative plane. For the profiles considered (Figs. $15,16,18$ ), velocity is scaled on the centreline axial velocity $\bar{u}_{c}$ and the radial coordinate is scaled on the local width $b$ of the external jet envelope. 
(a)

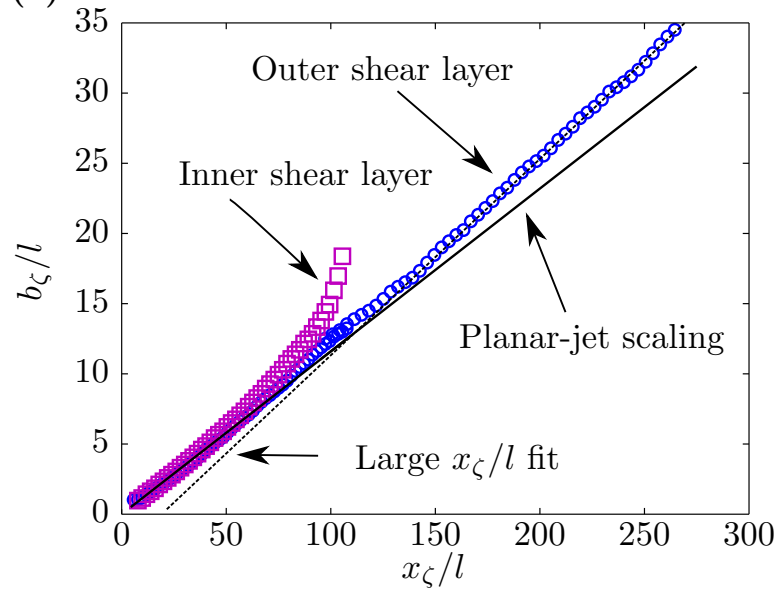

(b)

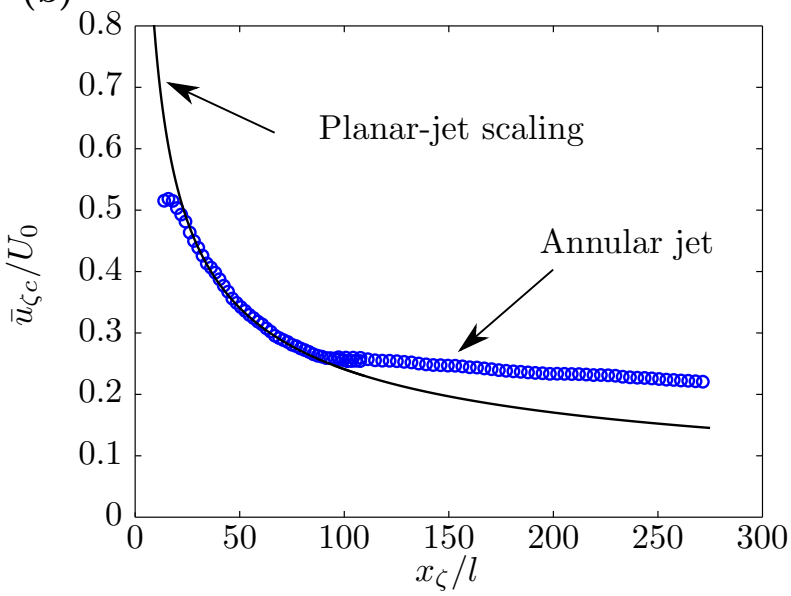

FIG. 14: (a) Normalised width of inner shear layer $b_{i n \zeta} / l(\square)$ and outer shear layer $b_{\text {out } \zeta} / l$ (o) versus streamwise distance $x_{\zeta} / l$ (see Fig. 6 ). The solid line with gradient 0.116 shows the linear shear layer growth for an idealised planar jet [25, p. 328]. The dashed line represents a linear best fit to our ventilated annular-jet data for $x_{\zeta} / l>150$ and has a gradient of 0.137 . (b) Streamwise centreline velocity for our ventilated annular jet $(\circ)$. The solid line plots the planar-jet velocity $\bar{u}_{\zeta c} / U_{0}=$ const. $\left(x_{\zeta} / l\right)^{-1 / 2}[25]$ with a constant of proportionality of 2.5 . For reference, the locations $x_{\zeta} / l=\{100,250\}$ are equivalent to $x / D_{o} \approx\{1.6,4.0\}$ on the longitudinal nozzle axis.

TABLE IV: Symbol with corresponding dimensionless axial distance $x / D_{o}$ downstream of the nozzle exit plane used in the velocity, turbulent intensity, Reynolds stress and turbulent viscosity profiles (Figs. 15, 16, 18a-b). Online: on travelling downstream, the colour of the symbols transmutes from blue to red.

\begin{tabular}{lllllllllllll}
\hline \hline Symbol & + & $\bigcirc$ & $*$ & $\bullet$ & $\times$ & $\square$ & $\diamond$ & $\triangleright$ & $\triangleleft$ & $\triangle$ & $\nabla$ & 文 \\
$x / D_{o}$ & 1 & 2 & 3 & 4 & 5 & 6 & 7 & 8 & 9 & 10 & 12 & 14 \\
\hline \hline
\end{tabular}

\section{Velocity 85 intensity profiles}

A visual inspection of the profiles of time-averaged axial velocity shown in Fig. 15(a), which extend to $x / D_{o}=14$ (recall that $D_{o} \approx 62 l$, Table I), suggests that a far-field state of approximate self-similarity is attained for $x / D_{o} \gtrsim 5$. Returning to Fig. 12(a) we note that the outer shear layer of the final profile ( $\hat{\xi}$ at $x_{\zeta}=265 l$, or, equivalently, $x \approx 4.5 D_{o}$ ) is also Gaussian and self-similar, suggesting that the outer shear layer transitions, without a break in self-similarity, into the single shear layer observed after the jet has merged.

For the profiles of time-averaged radial velocity, Fig. 15(b), there is no close collapse of the data, although for $x / D_{o} \gtrsim 7$ the profiles begin to more closely overlap. As noted in Sec. IIID, the cross-stream velocity $\bar{v}_{\zeta}$ profiles of the coalescing jet are not self-similar due to the influence of merging, and it was therefore to be expected that the radial velocity profiles $\bar{v}(x=$ const., $y$ ) of the coalesced jet appear to be approaching self-similarity downstream of the axial velocity profiles $\bar{u}(x=$ const., $y)$. Figure 15(b) clearly shows that the jet entrains fluid from the ambient (velocities towards the jet centreline in the ambient) and that fluid within the core of the jet moves outwards (velocities away from the centreline in the jet).

Dimensionless profiles of axial $\left(I_{u}=\sigma_{u} / \bar{u}_{c}\right)$ and radial $\left(I_{v}=\sigma_{v} / \bar{u}_{c}\right)$ turbulent intensities are 
(a)

(b)
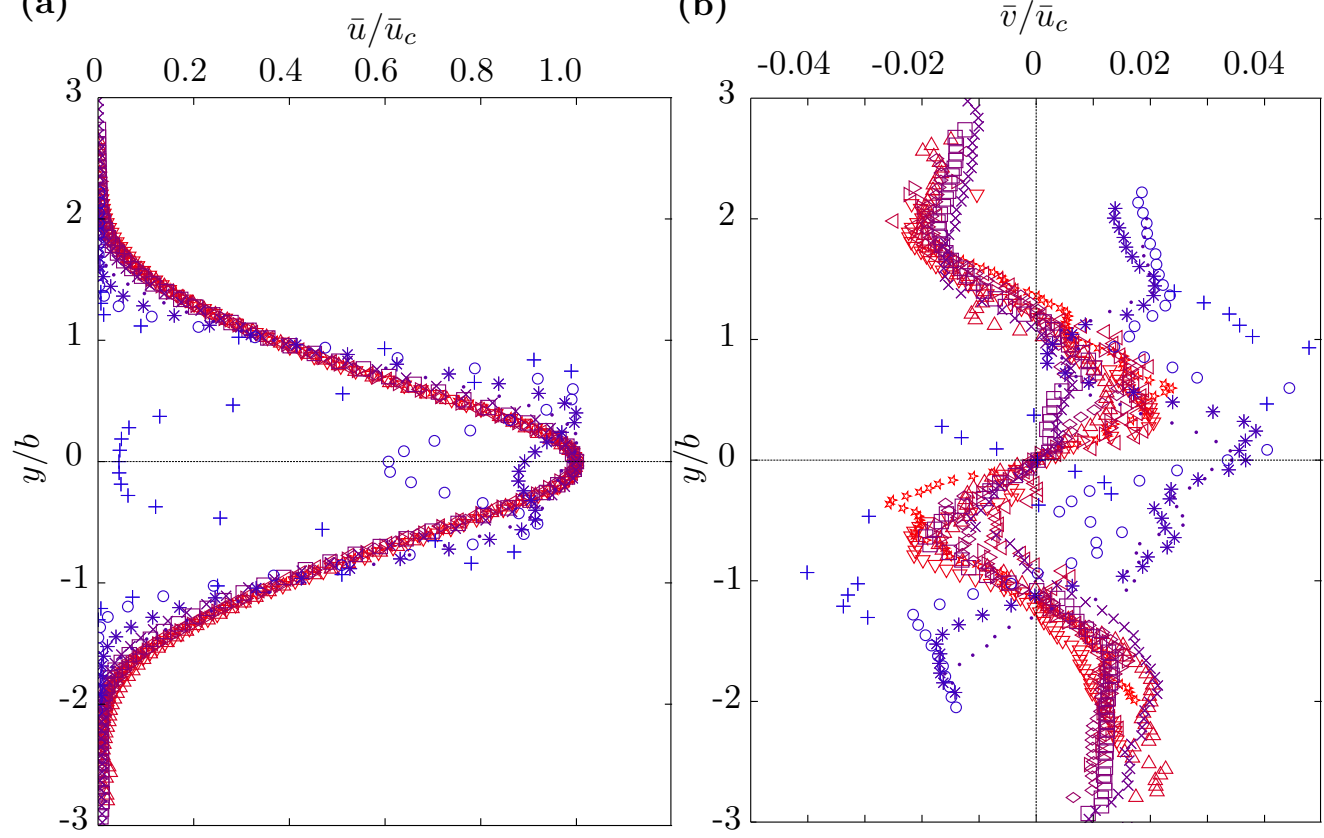

FIG. 15: Time-averaged velocity profiles for the ventilated annular jet: (a) axial velocity $\bar{u} / \bar{u}_{c}$ and (b) radial velocity $\bar{v} / \bar{u}_{c}$ at downstream distances from $x / D_{o}=1.0(+)$ to $x / D_{o}=14.0(\hat{s})$.

See Table IV for the downstream location corresponding to each symbol.

shown in Fig. 16. Although the profiles are qualitatively similar to those observed in round jets $[18,29]$, the peak turbulent intensities for the annular jet $\left(I_{u, \max }=0.24\right.$ and $I_{v, \max }=0.17$ at $x / D_{o} \approx 14$ ) are significantly lower (for the round jet of Hussein et al. [18] $I_{u, \max } \approx 0.29$ and $I_{v, \max } \approx 0.23$ ), at least for the downstream distances that were possible for us to make measurements. These differences indicate that the turbulence statistics do not reach a fully selfsimilar state within $16 D_{o}$ of the source. This claim is further supported by the results of Fig. 17 which show that the peak axial turbulent intensity $I_{u, \max }$ is not independent of $x / D_{o}$ within our measurement domain.

It is prudent here to check at what distance from the source the turbulent statistics reach self-similarity in round jets, and to compare this distance with the measurement domain of our experiment. To enable comparisons between round jets and annular jets we scale on the characteristic round-jet length scale $Q_{0} / M_{0}^{1 / 2}=\sqrt{A_{0}}$ [25]. Ferdman et al. [33] find self-similarity of the turbulence statistics in round jets at approximately 40 diameters downstream (for their nozzle $40 D \approx 45 \sqrt{A_{0}}$. . Ko and Chan [15] observed that the unventilated annular jet attained a self-similar state beyond approximately $5 D_{o}$ downstream (for their nozzle $5 D_{o} \approx 6.3 \sqrt{A_{0}}$ ). The corresponding measurements within our ventilated annular jet, even as far downstream as $15.7 D_{o}\left(\approx 70 \sqrt{A_{0}}\right)$, are not fully self-similar, suggesting that the open-core slender annular geometry and the resulting internal region of induced flow have a significant influence on the development of a jet.

Figure 18(a) shows normalised radial profiles of Reynolds stress $\overline{u^{\prime} v^{\prime}} / u_{c}^{2}$, a quantity which represents the turbulent shear stresses within the annular jet. As one would expect, the Reynolds stress profiles indicate that momentum is transferred away from the jet axis towards the ambient. Although the profiles appear to be progressing towards a self-similar state, similarity is not reached. These profiles, while qualitatively similar to the round-jet observations of Hussein et al. [18], exhibit marginally reduced peak Reynolds stresses $\left(\overline{u^{\prime} v^{\prime}} / u_{c}^{2}\right)_{\max } \approx 0.02$ (for round jets $\left.\left(\overline{u^{\prime} v^{\prime}} / u_{c}^{2}\right)_{\max } \approx 0.025\right)$ due to the aforementioned reduced peak turbulent intensities. These re- 
(a)

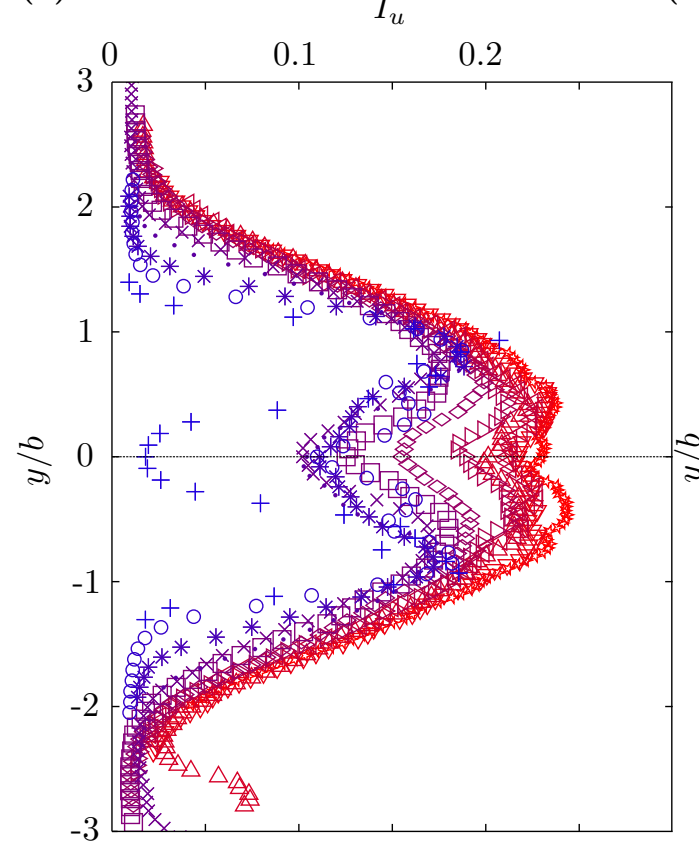

(b)

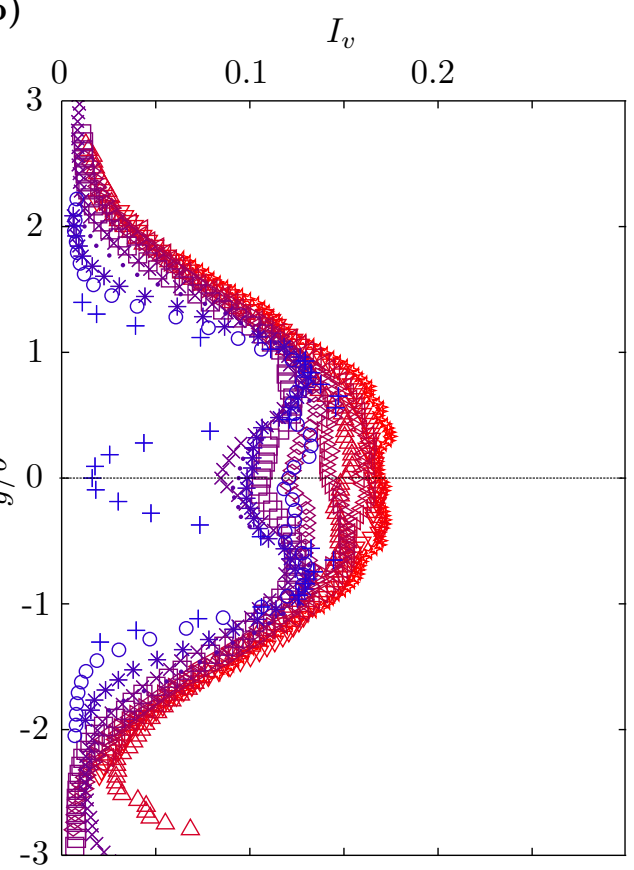

FIG. 16: Turbulent intensity profiles for the ventilated annular jet: (a) axial turbulent intensity $I_{u}(x, y)=\sigma_{u}(x, y) / \bar{u}_{c}(x)$ and (b) radial turbulent intensity $I_{v}(x, y)=\sigma_{v}(x, y) / \bar{u}_{c}(x)$. The downstream location corresponding to each symbol is given in Table IV.

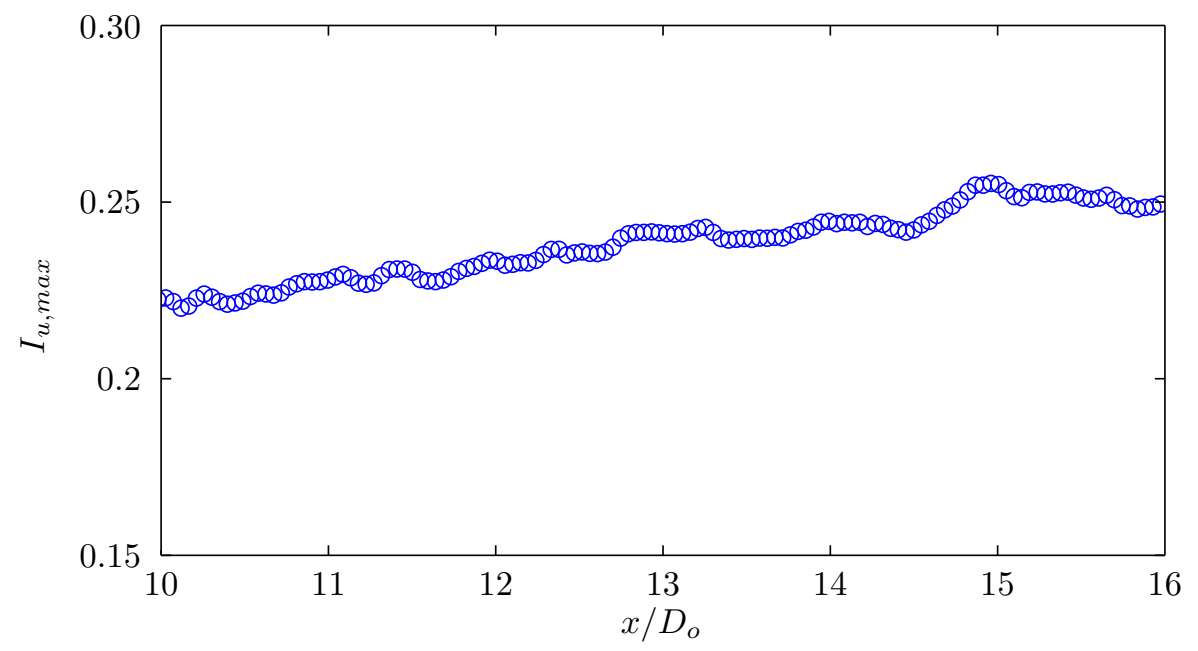

FIG. 17: Peak axial turbulent intensity $I_{u, \max }$ in the ventilated annular jet versus downstream distance $x / D_{o}$.

duced peak Reynolds stresses indicate that, within our measurement domain $0<x / D_{o}<16$, the magnitude of momentum transferred away from the jet centreline for the ventilated annular jet is less than for a fully developed round jet.

As we shall see below, evaluating the turbulent viscosity,

$$
v_{T}(x, y)=\frac{-\overline{u^{\prime} v^{\prime}}}{\partial \bar{u} / \partial y},
$$



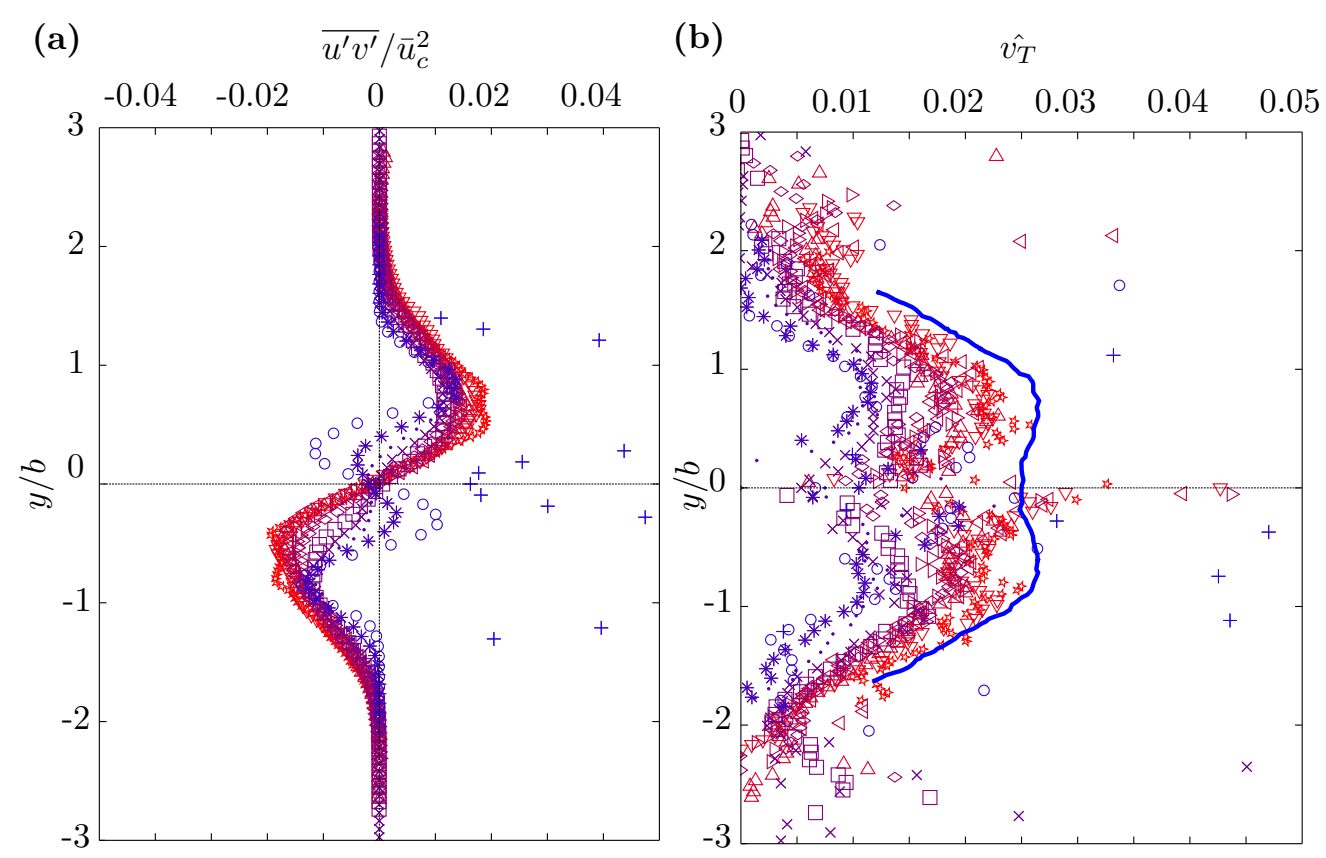

FIG. 18: Radial profiles of dimensionless: (a) Reynolds stress $\overline{u^{\prime} v^{\prime}} / \bar{u}_{c}^{2}$ and (b) turbulent viscosity $\hat{v}_{T}=v_{T} /\left(\bar{u}_{c} b\right)$. In (b) the line represents the fit of Hussein et al. [18] to their experimental data for a round jet. See Table IV for the downstream location corresponding to each symbol.

a measure of the radial momentum transport within the jet, lends support to the conclusion that the ventilated annular jet has a reduced momentum transport when compared to self-similar round jets, at least as far downstream as $x=16 D_{o}$. Radial profiles of normalised turbulent viscosity $\hat{v_{T}}=v_{T} /\left(\bar{u}_{c} b\right)$, Fig. 18(b), are qualitatively similar to those of Hussein et al. [18] for round jets (their results plotted as the continuous line) although with a reduced magnitude and, therefore, a reduced radial momentum transport. The profiles suggest that the momentum transfer occurs primarily for $|y / b|<1$, and reduces with distance from the jet centreline for $|y / b|>1$. The scatter near the jet axis occurs because the Reynolds stress is divided by a very small velocity gradient and thus small errors are amplified in this region. The implications of the reduced momentum transport are discussed in Sec. III E 2.

The development of radial momentum transport along the length of the jet becomes clear on plotting the axial variation in turbulent viscosity. This was achieved by calculating the dimensionless bulk turbulent viscosity $\left\langle\hat{v_{T}}\right\rangle$. The quantity $\left\langle\hat{v_{T}}\right\rangle$ represents an average of $\hat{v}_{T}$ across the jet and is calculated using the fit proposed by Ezzamel et al. [27], whereby a Gaussian velocity profile is assumed and the experimental profiles are fitted to the function

$$
F=2\left\langle\hat{v_{T}}\right\rangle \frac{y^{2}}{b^{2}} \exp \left(-\frac{y^{2}}{b^{2}}\right)
$$

Despite the uncertainty shown, Fig. 19 (०) indicates that $\left\langle\hat{v}_{T}\right\rangle$, and therefore radial momentum transport, increases with downstream distance.

Much of the work on the subject of entrainment by jets follows on from the classic work of Ricou and Spalding [34] in which they developed elegant experiments to satisfy the 'entrainment appetite' of the jet. Morton et al. [35] parameterise this entrainment by means of an entrainment constant $\alpha$, which links the radial velocity of the induced flow to the velocity along the jet centreline [35], 


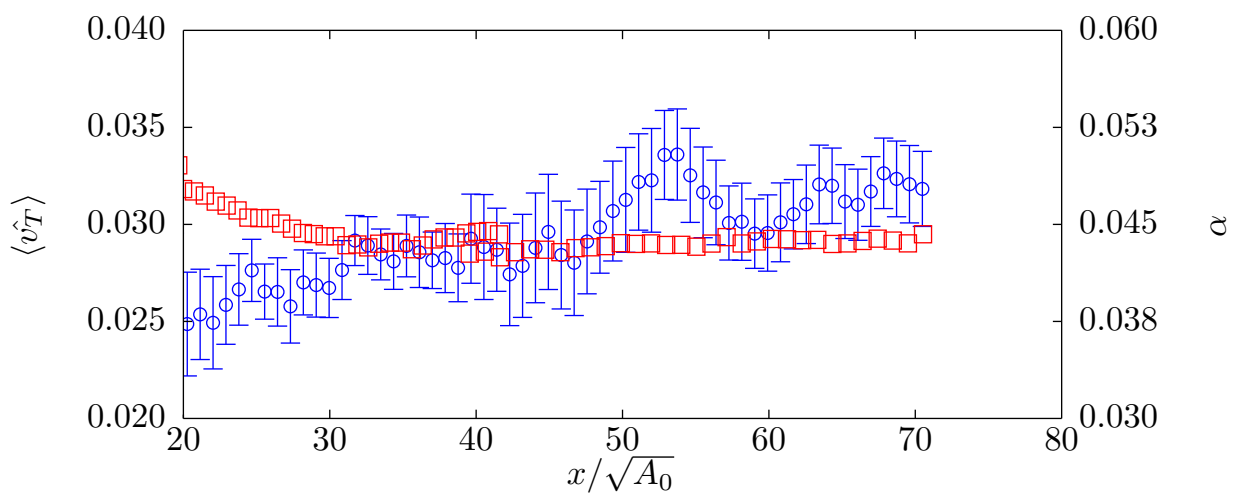

FIG. 19: Bulk turbulent viscosity $\left\langle\hat{v}_{T}\right\rangle$ (०) versus downstream distance $x / \sqrt{A_{0}}$. The error bars represent the $95 \%$ confidence interval obtained when fitting the data to (14). The entrainment constant $\alpha \approx(3 / 2)\left\langle\hat{v}_{T}\right\rangle$ [27], and as such, the axis on the right-hand side represents the conversion from $\left\langle\hat{v}_{T}\right\rangle$ to $\alpha$. Also plotted $(\square)$ is the entrainment constant worked out from (15).

TABLE V: Coefficients for (16a), (16b) and (16c) for the ventilated annular jet and the round jet.

\begin{tabular}{llll}
\hline \hline & $c_{1}$ & $c_{2}$ & $c_{3}$ \\
Ventilated annular jet & 0.102 & 7.5 & 0.231 \\
Round jet & 0.113 & 6.5 & 0.353 \\
Fischer et al. $[25]$ & 0.107 & 7.0 & - \\
Hussein et al. $[18]$ & - & 6.5 & - \\
\hline \hline
\end{tabular}

so that for the far field of our annular jet

$$
\frac{d Q}{d x}=2 \pi b \alpha \bar{u}(x, 0)
$$

Typically, the entrainment coefficient for jets falls within the range $0.045<\alpha<0.056$ [27]. Neglecting the contributions from higher order terms, from Ezzamel et al. [27], the entrainment constant $\alpha \approx(3 / 2)\left\langle\hat{v}_{T}\right\rangle$. Thus, the axis on the right of Fig. 19 shows that for $x / \sqrt{A_{0}} \gtrsim 50$ the estimates of $\left\langle\hat{v_{T}}\right\rangle$ give values of $\alpha$ that are consistent with those from the literature. Also plotted $(\square)$ are values of $\alpha$ calculated using (15) and values of $b, u(x, 0)$ and $d Q / d x$ from Fig. 20, where $d Q / d x$ has been estimated using the linear best fit line. For $x / \sqrt{A_{0}} \gtrsim 30$ these values of $\alpha$ coincide well with the estimates from the turbulent viscosity.

\section{Axial dependence}

To compare the far-field development of the annular jet with the classic round jet, we examine the axial dependence of their width $b$, axial velocity $\bar{u}(x, 0)$ and volume flux $Q$ (Fig. 20). For the round jet we performed independent PIV measurements as discussed in Appendix A. To enable direct comparisons, we once again scale the downstream distance on the characteristic round-jet length scale $\sqrt{A_{0}}[25]$. For our annular nozzle $D_{o} \approx 4.5 \sqrt{A_{0}}$.

Our data for the annular jet shown in Fig. 20(a-c) confirms that, as for a round jet, far from the nozzle, jet width, inverse axial velocity, and volume flux increase linearly with $x$ and can be described by 


$$
b=c_{1}\left(x-x_{0}\right), \quad(16 \mathrm{a}) \quad \bar{u}(x, 0)=c_{2} U_{0}\left(\frac{x-x_{0}}{\sqrt{A_{0}}}\right)^{-1}, \quad(16 \mathrm{~b}) \quad Q(x)=c_{3} Q_{0} \frac{x-x_{0}}{\sqrt{A_{0}}},
$$

where $x_{0}$ denotes the virtual origin (see Sec. IIIE3). Estimates of volume flux were calculated using (12). The constants $c_{1}, c_{2}$ and $c_{3}$ describing the far-field behaviour of the round and annular jets are given in Table V. Although from these entries it would appear that the decay rate $\left(c_{2}\right)$ of the annular jet velocity exceeds that of our round jet (and the round jets of Hussein et al. [18] and Fischer et al. [25]), the turbulent quantities within our measurement domain have not reached self-similarity and, therefore, the decay rate would likely evolve further with downstream distance. The increasing turbulent intensities and turbulent viscosity with distance downstream, reported in Sec. III E 1, indicate an increasing radial momentum transfer, which will result in a reducing decay constant (a larger decay constant $c_{2}$ signifying a lower decay rate) and an increasing entrainment rate $c_{3}$ in the annular jet.

Nearer to the nozzle $\left(x / \sqrt{A_{0}} \lesssim 40\right)$, the volume flow rates of the annular and round jets differ significantly. This difference is in part an artefact of (12) which, for the annular jet, includes the contribution of the internal induced flow in $Q(x)$, a contribution that is not strictly part of the jet but rather of the induced flow. To exclude the internal induced-flow region in our comparisons of annular- and round- jet flow rates, we may compare flow rates at the merge point. The volume flux of the annular jet at the merge point (located at either $5.0 \sqrt{A_{0}}\left(\approx 1.11 D_{o}\right)$ or $7.2 \sqrt{A_{0}}\left(\approx 1.60 D_{o}\right)$, see Sec. III B) exceeds that of the round jet by $200-210 \%$ (Fig. 20c), suggesting that entrainment of this internal induced flow serves to increase the volume flux of the annular jet near the nozzle. Estimates of the volume flux $\left(Q / Q_{0}\right)$ very near the source $\left(x / \sqrt{A_{0}} \lesssim 1.5\right)$ are inaccurate due to a combination of the large velocity gradients and the spatial resolution of the data.

For the ventilated annular jet, all three quantities $\left(b / \sqrt{A_{0}},\left(\bar{u}(x, 0) / U_{0}\right)^{-1}, Q / Q_{0}\right)$ attain an approximately linear behaviour for $x / \sqrt{A_{0}} \gtrsim 40$; for the round jet we estimate for $x / \sqrt{A_{0}} \gtrsim 20$. This difference is attributed to the near-field planar-jet region and the distance over which the annular jet coalesces prior to asymptoting to round-jet behaviour.

\section{A note on virtual origins}

The position of the virtual origin $x=x_{0}$ for the ventilated annular jet proved to be sensitive to the downstream distance at which we assumed the far-field linear behaviour (16a-c) to commence. As discussed earlier, we believe this stems from the fact that the jet has not yet reached a fully self-similar state. There are, however, trends of note. The annular nozzle geometry shifts the virtual origin upstream relative to both the round jet and the nozzle itself. From fitting data in the range $40<x / \sqrt{A_{0}}<70$, we locate the virtual origin at $x_{0} / \sqrt{A_{0}}=-4.0 \pm 0.9$, where \pm 0.9 represents the variation in the virtual origin between the different quantities considered. This upstream shift occurs due to the relatively large nozzle diameter $D_{o}$ when compared with the slot width $l$.

\section{F. Transition $\left(1 \lesssim x / D_{o} \lesssim 10\right)$}

Between the near-nozzle region, where the jet exhibits planar-jet behaviour $\left(x_{\zeta} / l<60\right.$ or, equivalently, $x / D_{o}<1$, Sec. IIID), and the far field, where the jet exhibits round-jet behaviour $\left(x / \sqrt{A_{0}}>45\right.$ or, equivalently, $x / D_{o}>10$, Sec. III E), the flow may be regarded as in transition between these two states. The profiles of time-averaged streamwise $\bar{u}_{\zeta}\left(x_{\zeta}=\right.$ const., $\left.y_{\zeta}\right)$ and axial $\bar{u}(x=$ const., $y)$ velocity in the outer shear layer (for $y_{\zeta} / b_{\zeta} \geq 0$ in Fig. $12 \mathrm{a}$ and $x / D_{o} \geq 5$ in 
(a)

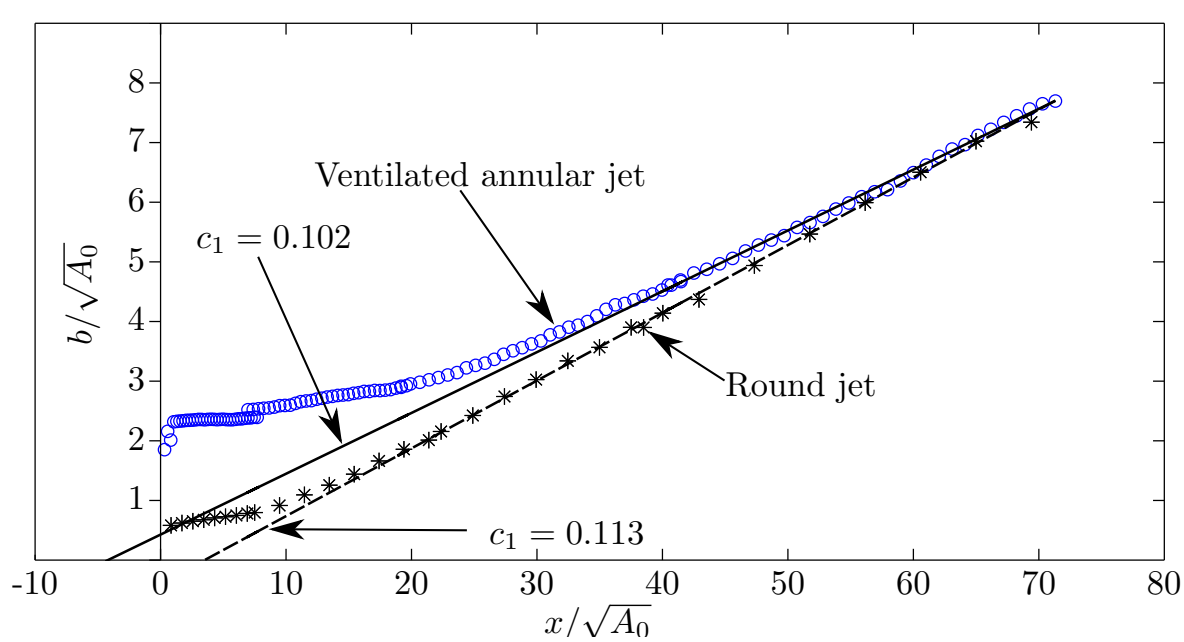

(b)

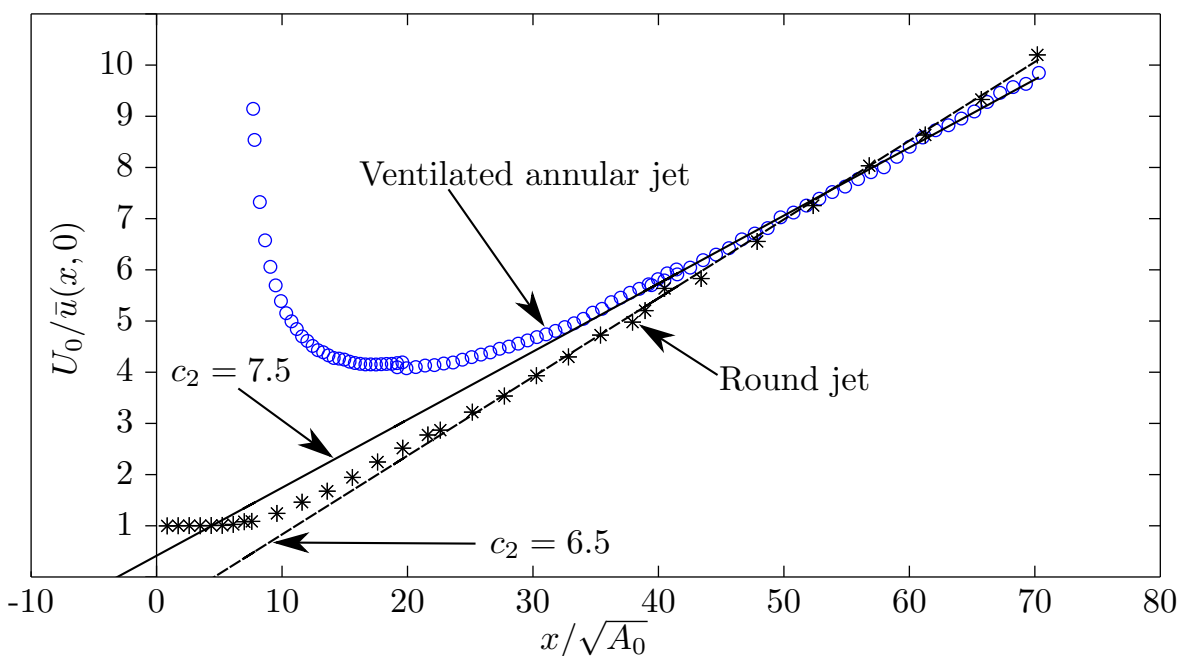

(c)

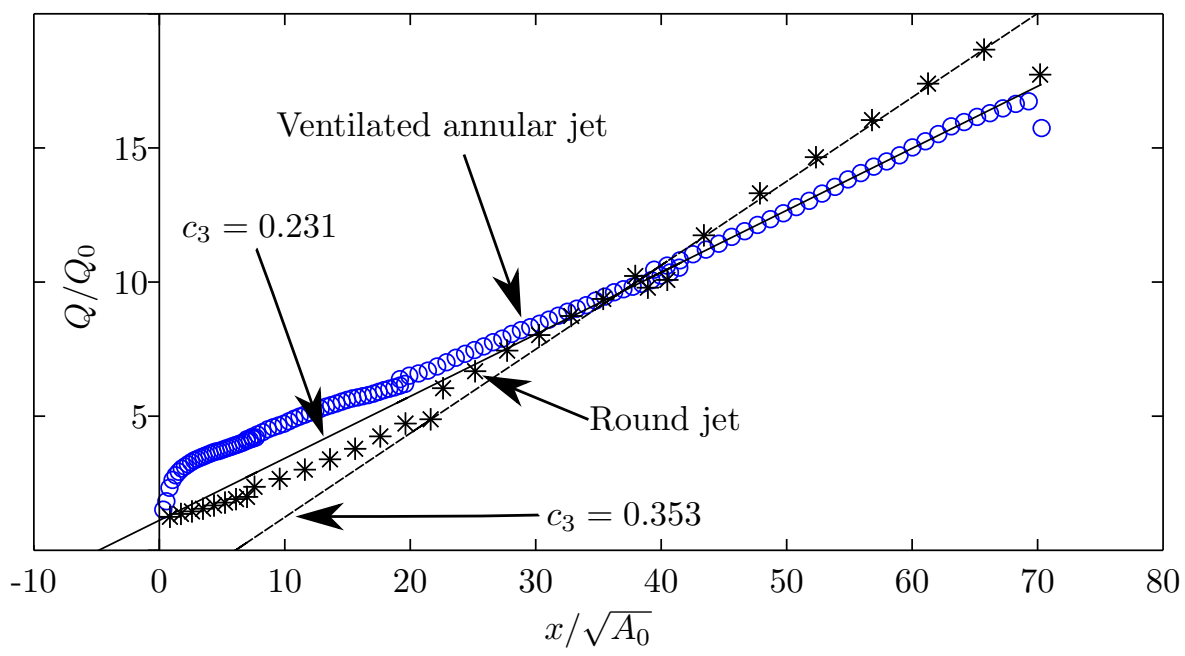

FIG. 20: Dimensionless variation with distance downstream $x / \sqrt{A_{0}}$ of: (a) jet width $b / \sqrt{A_{0}}$; (b) inverse axial velocity $\left(\bar{u}(x, 0) / U_{0}\right)^{-1}$; and (c) volume flux $Q / Q_{0}$ (calculated using (12)).

Ventilated annular jet data ( $\circ$ ) and best linear fit to this data for $x / \sqrt{A_{0}} \geq 40$ (solid line). Round-jet data $(*)$ and best linear fit to this data for $x / \sqrt{A_{0}} \geq 20$ (dashed line) as measured in our validation experiments (see Appendix A for details). The best fit lines are given by (16a), (16b), (16c) with the constants $c_{1}, c_{2}$, and $c_{3}$ given on the figures. The virtual origin $x_{0}$ is the location at which the best fit lines cross the $x$-axis. 
Fig. 15a) remain self-similar throughout this transitional region, suggesting that the influence of the coalescence on the structure of the outer shear layer is small. However, the absence of full self-similarity in the profiles of the time-averaged cross-stream $\bar{v}_{\zeta}\left(x_{\zeta}=\right.$ const., $\left.y_{\zeta}\right)$ and radial $\bar{v}(x=$ const., $y)$ velocities (Figs. $12 \mathrm{~b}$ and $15 \mathrm{~b}$ ) demonstrates that the jet is evolving dynamically along our entire domain of measurement. It is within the transitional region that the high-velocity centreline extending from the annular slot merges. Beyond the reattachment point, located at $4.5 D_{o}$ downstream, the jet can be regarded as having completely coalesced; the outer shear layer has absorbed the entirety of the inner shear layer, although the flow continues to develop downstream (Sec. III E).

Clear indicators for the transition are also evident in the turbulent intensity profiles, where a local self-similarity is observed near the nozzle (see profiles in Fig. 13 for $30 \leq x_{\zeta} / l \leq 105$ or, equivalently, $0.5 \leq x / D_{o} \leq 1.75$ ) and a second local self-similarity is evident far downstream (Fig. 16). Between these regions, the turbulent intensities within the inner shear layer tend to decrease as the jet merges (see $y_{\zeta} / l \leq 0$ on profiles in Fig. 13 for $x_{\zeta} / l \geq 105$ ) as a result of the reduced velocity gradient in the inner shear layer (see profiles in Fig. 12 for $x_{\zeta} / l \geq 105$ ). Once the inner shear layer has been absorbed, the turbulent intensities begin to increase (see profiles in Fig. 16 for $\left.x / D_{o} \geq 5\right)$. The transition is also noted on examining the variation of the jet width and centreline velocity with distance downstream (Figs. 14, 20). The regions of planar-jet behaviour and of local self-similarity near the nozzle terminate at the same location $\left(x_{\zeta} / l \approx 100\right)$ due to the link between the jet development and its turbulent properties. We anticipate that the extents and characteristics of the transitional region to be primarily influenced by the inner $D_{i}$ and outer $D_{o}$ diameters of the nozzle, as these length scales characterise the separation between the opposite sides of the (inner and outer) shear layers and of the jet centreline at the source.

\section{CONCLUSIONS}

The dynamics of a turbulent jet produced on ejecting fluid steadily through a slender annular slot surrounding an open core into an otherwise quiescent uniform environment have been studied. Using high-resolution velocity measurements obtained from particle image velocimetry we have identified four regions of flow, each characterised by a distinct behaviour and dominant length scale (these regions and their approximate extents are illustrated on Fig. 2). Herein we targeted a slender annulus, the ratio of internal to external diameters $D_{i} / D_{o}=0.968$ being close to the theoretical idealised limit of $D_{i} / D_{o}=1$. Moreover, the core was almost fully open with a ventilation ratio of $D_{v} / D_{i} \approx 0.9$.

Unique to the open-core, or ventilated, annular geometry is an internal region of induced flow that is bounded by the jet itself. This bounded induced-flow region is driven by entrainment into the inner shear layer of the jet and its extent characterised by the internal diameter $D_{i}$. Our measurements indicate that the volume flux entrained into the jet from this internal flow region is comparable with the source volume flux $\left(\approx 1.2 Q_{0}\right)$ and, as a consequence, near-field dilution is enhanced compared with a classic round jet. This feature could potentially be put to good effect in applications where there is a need, or benefit, to rapidly dilute the source fluid. For example, effluent discharges into the ocean often take the form of a round jet [25] and dilution could be significantly increased through the use of a slender ventilated annular nozzle geometry. Given the near-idealised geometry considered, the aforementioned volume flux induced through the core represents a practical upper limit, this flux reducing as the ventilation ratio $D_{v} / D_{i}$ decreases.

The annular jet itself consists of a near-field planar-jet-like region, a transitional region and, ultimately, a far-field round-jet region. Within the planar-jet region, the behaviour of the flow is characterised by the slot width $l=\left(D_{o}-D_{i}\right) / 2$; our results suggest that the jet has no knowledge 
of the annular nature of the source until merging begins. Far downstream, we observe behaviour characteristic of a round jet, namely a linear spreading rate, a linear decay of inverse axial velocity and a linear increase in volume flux. Based on comparisons made with classic round jets it appears that the relevant length scale of this far-field region is the square root of the slot area $\sqrt{A_{0}}$. While there is approximate self-similarity of the time-averaged axial velocities, the turbulent quantities continue to evolve with downstream distance indicating true self-similarity has not yet been achieved despite the scale of our measurement domain extending to $16 D_{o}$. Nonetheless, it is clear that existing scalings for planar jets and round jets apply to the near-field and far-field regions respectively. With respect to the far field, the annular jet may be replaced with a round jet at the virtual origin - our results suggest the origin is located at $x / \sqrt{A_{0}}=-4.0$.

\section{ACKNOWLEDGMENTS}

The authors GRH and SP gratefully acknowledge the financial support of Dyson Technology Ltd and the EPSRC Industrial Case Award programme 13440009. We would also like to express our sincere thanks to Tim Jukes, Jimmy Lirvat, Frederic Nicolas, Ludovic Desvard and Charles Collis for many interesting discussions on the subject. Finally, our thanks to Thomas Blower for his technical input on the nozzle design.

[1] S. Padhani and G. R. Hunt, "The coalescence of a turbulent slender open-core annular jet - the role of the diameter ratio," Phys. Rev Fluids (In preparation).

[2] N. W. M. Ko, "Annular jets of small inner diameter," J. Sound Vib. 68, 468-472 (1980).

[3] N. W. M. Ko, "Annular jets of large inner diameter," J. Sound Vib. 69, 616-621 (1980).

[4] N. W. M. Ko and K. C. Leung, "Covariance measurements in the initial region of an annular jet," J. Sound Vib. 80, 339-354 (1982).

[5] N. W. M. Ko, "Excited annular jets of large inner diameter," J. Sound Vib. 88, 576-578 (1983).

[6] N. W. M. Ko and K. M. Lam, "Further measurements in the initial region of an annular jet," J. Sound Vib. 92, 333-348 (1984).

[7] N. W. M. Ko and K. M. Lam, "Flow structures of a basic annular jet," AIAA J. 23, 1185-1190 (1985).

[8] K. M. Lam, N. W. M. Ko, and K. K. Lau, "Wake and wake-induced shear-layer excitation in an annular jet," Phys. Fluids 29, 3121-3134 (1986).

[9] K. M. Lam and N. W. M. Ko, "Investigation of flow structures of a basic annular jet," AIAA J. 24, 1488-1493 (1986).

[10] K. K. Lau and N. W. M. Ko, "On the formation of wake induced structures in a basic annular jet," in 11th Australasian Fluid Mechanics Conference (University of Tasmania, Hobart, Australia, 1992) pp. $383-387$.

[11] N. W. M. Ko, K. K. Lau, and K. M. Lam, "Dynamics of interaction modes in excited annular jets," Experimental Thermal and Fluid Science 17, 319-338 (1998).

[12] R. Mason, F. Nicolas, and R. Pitt, "Bladeless is more," ANSYS Advantage 6, 5-7 (2010).

[13] H. Li, H-S. Deng, and Y-B. Lai, "Numerical and experimental research on the outlet flow field for the air multiplier," Applied Thermal Engineering 93, 652-659 (2016).

[14] N. A. Chigier and J. M. Beer, "The flow region near the nozzle in double concentric jets," Journal of Basic Engineering 86, 797-804 (1964).

[15] N. W. M. Ko and W. T. Chan, "Similarity in the initial region of annular jets: three configurations," J. Fluid Mech. 84, 641-656 (1978).

[16] N. W. M. Ko and W. T. Chan, "The inner regions of annular jets," J. Fluid Mech. 93, 549-584 (1979).

[17] W. T. Chan and N. W. M. Ko, "Coherent structures in the outer mixing region of annular jets," J. Fluid Mech. 89, 515-533 (1978). 
[18] H. J. Hussein, S. P. Capp, and W. K. George, "Velocity measurements in a high-Reynolds-number, momentum-conserving, axisymmetric, turbulent jet," J. Fluid Mech. 258, 31-75 (1994).

[19] K. Li and R. S. Tankin, "A study of cold and combusting flow around bluff-body combustors," Combustion Science and Technology 52, 173-206 (1987).

[20] H. A. Warda, S. Z. Kassab, K. A. Elshorbagy, and E. A. Elsaadawy, "An experimental investigation of the near-field region of free turbulent round central and annular jets," Flow Measurement and Instrumentation 10, 1-14 (1999).

[21] H. Rehab, E. Villermaux, and E. J. Hopfinger, "Flow regimes of large-velocity-ratio coaxial jets," J. Fluid Mech. 345, 357-381 (1997).

[22] G. K. Batchelor, An Introduction to Fluid Dynamics (Cambridge University Press, Cambridge, 1967).

[23] P. E. Dimotakis, "The mixing transition in turbulent flows," J. Fluid Mech. 409, 69-98 (2000).

[24] W. M. Haynes, ed., CRC Handbook of Chemistry and Physics, 93rd ed. (CRC Press, Boca Raton, 2012).

[25] H. B. Fischer, E. J. List, R. C. Y. Koh, J. Imberger, and N. H. Brooks, Mixing in Inland and Coastal Waters, 1st ed. (Academic Press, New York, 1979).

[26] LaVision, Product manual for DaVis 8.2 (LaVision GmbH, Item No. 1105011-4, Gottingen, Germany, 2014).

[27] A. Ezzamel, P. Salizzoni, and G. R. Hunt, "Dynamical variability of axisymmetric buoyant plumes," J. Fluid Mech. 765, 576-611 (2015).

[28] H. Schlichting, Boundary-layer theory (McGraw-Hill, New York, 1968).

[29] H. Wang and A.W-K. Law, "Second-order integral model for a round turbulent buoyant jet," J. Fluid Mech. 459, 397-428 (2002).

[30] W.R. Quinn, "Upstream nozzle shaping effects on near field flow in round turbulent free jets," Eur. J. Mech. B Fluids 25, 279-301 (2006).

[31] A. Krothapalli and D. Baganoff, "On the mixing of a rectangular jet," J. Fluid Mech. 107, 201-220 (1981).

[32] F. O. Thomas and V. W. Goldschmidt, "Structural characteristics of a developing turbulent planar jet," J. Fluid Mech. 163, 227-256 (1986).

[33] E. Ferdman, M. V. Otugen, and S. Kim, "Effect of initial velocity profile on the development of round jets," Journal of Propulsion and Power 16, 676-687 (2000).

[34] F. P. Ricou and D. B. Spalding, "Measurements of entrainment by axisymmetrical turbulent jets," J. Fluid Mech. 11, 21-32 (1961).

[35] B. R. Morton, G. Taylor, and J. S. Turner, "Turbulent gravitational convection from maintained and instantaneous sources," Proceedings of the Royal Society A: Mathematical, Physical and Engineering Sciences 234, 1-23 (1956).

[36] T. Jukes, J. Lirvat, F. Nicolas, S. Padhani, and G. R. Hunt, "Flow control using annular jets," in European Drag Reduction and Flow Control Meeting (Cambridge, UK, 2015).

[37] M. Raffel, C. E. Willert, S. T. Wereley, and J. Kompenhans, Particle Image Velocimetry, 2nd ed. (Springer, Berlin, 2007) p. 448.

[38] N. A. Chigier and J. M. Beer, "Velocity and static-pressure distributions in swirling air jets issuing from annular and divergent nozzles," Journal of Basic Engineering 86, 788-796 (1964).

[39] M. Vanierschot, K. Van Dijck, P. Sas, and E. Van Den Bulck, "A study of the wake dynamics of annular jet flows with and without swirl," in 17th International Symposium on Applications of Laser Techniques to Fluid Mechanics (Lisbon, Portugal, 2014).

\section{Appendix A: Benchmarking PIV experiments}

This appendix overviews the 'benchmarking' PIV experiments that were performed to ensure confidence in the measurements obtained herein; for further information, see Jukes et al. [36]. The round-jet experiment of Hussein et al. [18] and data therein was taken as the benchmark, and their experiment repeated as closely as possible using our PIV system. Our resulting round-jet data is compared with the ventilated annular jet in Sec. III. 


\section{Round-jet experimental set-up}

The round-jet nozzle used was almost identical to that of Hussein et al. [18], with a diameter $D=25.4 \mathrm{~mm}, 121: 1$ contraction ratio and upstream flow conditioning consisting of a honeycomb and three mesh screens. The jet issued from the nozzle (supplied by a variac controlled centrifugal fan) with an approximately top-hat velocity profile and low initial turbulence, at a Reynolds number of $\operatorname{Re}_{D}=U_{0} D / v \approx 95000$. The experimental environment and procedure were identical to that described in Sec. II, with 500 PIV velocity fields acquired in order to estimate the time-averaged quantities and turbulence statistics of interest.

\section{Near field}

Planar PIV results were compared to Pitot tube measurements immediately adjacent to $(x / D=$ $0)$ and slightly downstream $(x / D=0.6)$ of the circular orifice. The PIV velocities agreed well with those from the Pitot measurements, with a difference of $\lesssim 2 \%\left(\approx 1 \mathrm{~m} \mathrm{~s}^{-1}\right)$. This small difference in velocities could potentially be related to errors in the Pitot tube measurements, including errors in the pressure transducer, Pitot alignment, blockage effect and uncertainty in the air density estimated from thermocouple measurements. From our PIV results we estimated a boundary-layer thickness of $0.85 \mathrm{~mm}$, similar to the value of $0.7 \mathrm{~mm}$ measured by Hussein et al. [18] using a hotwire.

Downstream of the nozzle, the approximately uniform exit profile of the jet was penetrated by the shear layer that forms at the jet edge, with a potential core extending for $5-6 D$ from the source, again in close agreement with Hussein et al. [18]. Beyond 5-6D, the flow was fully turbulent.

\section{Far field}

To further validate the PIV system, our far-field round-jet PIV measurements were compared with those of Hussein et al. [18] and of Ricou and Spalding [34]. The centreline velocity $\bar{u}_{c}$ and spreading rate of the $\bar{u}_{c} / 2$ velocity contour (i.e. jet half width $b_{0.5}$ ) were within $\pm 2 \%$ and $\pm 2.5 \%$, respectively, of the results reported by Hussein et al. [18] who give $U_{0} / \bar{u}_{c}=(1 / 5.8)(x / D-4)$ and $b_{0.5} / D=0.094(x / D-4)$.

Hussein et al. [18] did not measure the volume flux and so comparisons were made with the measurements of Ricou and Spalding [34]. Estimates of volume flux obtained far from the nozzle (by integrating the velocity profiles around the streamwise axis) were compared with, and a good agreement (within $-7 \%$ and $+2 \%$ ) was found with, the values predicted by the fit of Ricou and Spalding [34], namely, $Q / Q_{0}=0.32 x / D$.

A full analysis of the time-averaged velocity profiles and second-order turbulence statistics was also carried out. Hussein et al. [18] observed self-similarity of the time-averaged profiles at approximately $30 D$ downstream of the nozzle, a result supported by our PIV measurements. While our turbulent intensity measurements are qualitatively similar to those of Hussein et al. [18], the values are marginally lower $(\sim-5 \%)$. Several authors have noted that the turbulent intensities do not become self-similar until far from the nozzle. The measurements of Hussein et al. [18] were taken further downstream $(x / D=70)$ than our planar PIV measurements $(x / D=55)$, which may explain the discrepancy with the turbulent intensities we measure. This explanation is supported by the continuing evolution of the turbulent intensities within our round jet with downstream distance $x$.

In conclusion, our PIV results for the round jet match very well with the results of Hussein et al. [18] and Ricou and Spalding [34], indicating that the PIV system was performing well, giving 
us confidence in our measurements of the annular jet.

\section{Appendix B: PIV processing}

PIV makes use of cross-correlation techniques, on a sequential image pair, to calculate the velocity vectors from the bulk motion of particles within the intensity fields; see Raffel et al. [37] for details. The images of the seeded flow were processed and velocity vectors calculated using LaVision's DaVis 8.2.1 software as follows. Images were pre-processed with an 8 pixel sliding background subtraction, increasing the signal-to-noise ratio. Following this, a multi-pass cross-correlation algorithm was used, with one pass using an interrogation window size of $64 \times 64$ pixels, and then two passes at a size of $32 \times 32$ pixels with a $50 \%$ overlap. A 'standard' PIV algorithm (via FFT, no zero-padding) was used for the first pass, and the second two passes used a 'normalised' algorithm in 'high accuracy' mode. The first two passes were post-processed by removing spurious vectors using a two-stage median filter and filling empty vectors using interpolation. Post-processing was not applied to the final pass. For the planar PIV, the final passes were weighted using a 2:1 elliptical Gaussian weighting function along the $x$-axis (since the flow is predominantly axial). The velocity vectors were then imported into MATLAB for analysis. Small vertical $(<10 \mathrm{~mm})$ and rotational $\left(<2^{\circ}\right)$ corrections were made to align the central streamline with the source symmetry axis.

\section{Appendix C: Quality control}

To ensure that peak-locking effects remained minimal, we chose appropriate window sizes following Raffel et al. [37] and then analysed the resulting data within the LaVision DaVis software to confirm that sub-pixel precision was maintained. Misalignment of the camera and laser-sheet would result in errors due to out-of-plane particle movement and errors when translating from pixel displacement to a physical displacement. This error was quantified using the average deviation given by the DaVis software during the calibration procedure. The average deviations for the various fields of view are given in Table VI; according to LaVision [26], values below 1 pixel are considered to be good, and thus we can infer that the error due to misalignment was negligible. The data obtained from PIV represents a spatial average over the interrogation window and, as such, errors are introduced when considering the spatial derivatives of the velocity field.

Following Raffel et al. [37], algorithm errors were quantified by shifting an existing image of a single particle field by a known amount, then carrying out PIV on the shifted image pair. The pixel movement calculated by the PIV algorithm can then be compared with the known pixel movement. This calculation gives us two components of the error - a systematic bias error $\left(\epsilon_{\text {bias }}\right)$ and a RMS error $\left(\epsilon_{R M S}\right)$ - as given in Table VI. Both components of the algorithm error were small, never exceeding $1.3 \%$.

\section{Appendix D: Symmetry}

To assess whether the nozzle produced an axisymmetric jet, and thus that data collected from the chosen $x-y$ plane could be regarded as representative of the jet as a whole, we analysed the circumferential variation of the time-averaged axial velocity $\bar{u}(x=$ const., $r, \theta)$, obtained using stereoscopic PIV on a plane perpendicular to the nozzle axis and located relatively close to the source (at $\left.x=0.5 D_{o}\right)$. 
TABLE VI: The average deviation and algorithm errors in the four PIV measurement domains and at the nozzle exit (cf. Fig. 5 and Table II).

\begin{tabular}{lccc}
\hline \hline & \multicolumn{2}{c}{ Pixel movement error } & Average deviation \\
\hline FOV & $\epsilon_{\text {bias }}$ & $\epsilon_{R M S}$ & (pixels) \\
\hline NE & $0.015 \%$ & $0.178 \%$ & 0.206 \\
1 & $0.001 \%$ & $0.107 \%$ & 0.642 \\
2 & $-0.004 \%$ & $0.071 \%$ & 0.614 \\
3 & $-0.006 \%$ & $0.087 \%$ & 0.392 \\
4 & $-0.019 \%$ & $1.200 \%$ & 0.283 \\
\hline \hline
\end{tabular}

The contour plot in Fig. 21(a) shows the variation of $\bar{u}(r, \theta)$ across this section of flow. A series of local minima in the velocity are evident, at $22-23^{\circ}$ intervals (approx.), and coincide with the location of the flow-straightening vanes. Figure 21(a) indicates that the peak velocities are located on the perimeter of a circle with radius $r / D_{o}=0.462$. Figure 21(b) plots the circumferential variation in $\bar{u}\left(r / D_{o}=0.462, \theta\right)$ for $0 \leq \theta \leq 2 \pi$, i.e. around this high-velocity central ring of the jet. While highlighting the influence of the flow straighteners, these measurements reveal that the standard deviation of the peak velocity $\sigma_{p} / U_{0}(=0.031)$ is within $10 \%$ of the mean peak velocity $\bar{u}_{p}$. Therefore, the influence of the near-source flow asymmetries on the velocity measurements is expected to be relatively small. This is supported by the observation that the standard deviation of the velocity fluctuations along this high-velocity central ring (see dashed line on Fig. 21b) is over twice the size of the standard deviation of the peak velocity $\left(\sigma_{u}\left(r / D_{o}=0.462, \theta\right) / \sigma_{p} \approx 2.2\right)$. These small asymmetries indicate a weak $\theta$-dependence close to the source. Moving downstream, we anticipate the asymmetries appearing in time-averaged quantities will reduce further, due to the mixing and merging of the jet. Estimates of the momentum integral (discussed in Sec. III A), calculated from the $x-y$ measurement plane, appear to indicate a lack of momentum integral conservation near the nozzle and a conserved momentum integral further downstream lending support to the notion that the asymmetries reduce as the jet mixes and merges.

To establish whether the circumferential velocity component is significant, a measure of the degree of swirl within the jet was also estimated from the PIV measurements taken on the crosssection.

Chigier and Beer [38] compare time-averaged circumferential and streamwise momentum fluxes by means of a dimensionless swirl number $S$, defined as

$$
S=\frac{\int_{0}^{\infty} \int_{0}^{2 \pi} \bar{u} \bar{u}_{\theta} r \mathrm{~d} \theta \mathrm{d} r}{\int_{0}^{\infty} \int_{0}^{2 \pi} \bar{u}^{2} r \mathrm{~d} \theta \mathrm{d} r} .
$$

A value of $S=0$ corresponds to a section without swirl. Vanierschot et al. [39] regard a value of $|S|>0.12$ as corresponding to swirling flows. We obtain a swirl number of $|S|=0.0030$, i.e. two orders of magnitude less than the Vanierschot et al. [39] swirl number, from which we assert that the time-averaged circumferential velocity is negligible and affirm that we can suitably capture the local time-averaged behaviour of the jet using planar PIV in the chosen $x-y$ plane. 
(a)
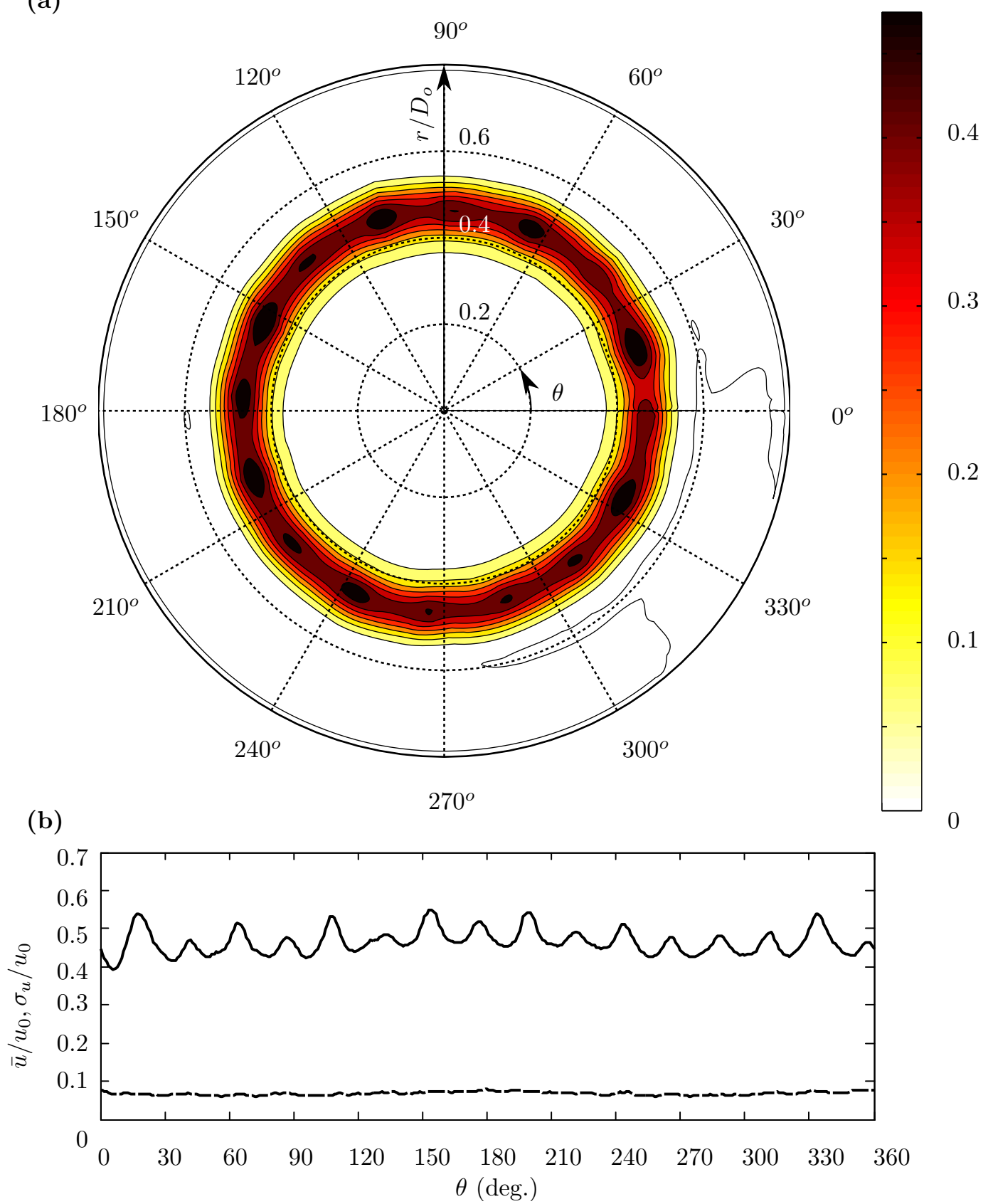

FIG. 21: Cross-section through the ventilated annular jet at $x / D_{o}=0.5$ (for $D_{i} / D_{o}=0.968$ and $R e_{s}=3200$ ). (a) Contour plot of time-averaged axial velocity $\bar{u}(r, \theta)$. The colour bar indicates the non-dimensional axial velocity $\bar{u} / U_{0}$ (b) Solid line: the circumferential variation of the time-averaged axial velocity on the circle $\left(r / D_{o}=0.462,0 \leq \theta \leq 2 \pi\right)$, indicating the high-velocity central ring of the jet. Dashed line: the standard deviation $\sigma_{u}\left(r / D_{o}=0.462, \theta\right)$ of these velocities. The average velocity for $0 \leq \theta \leq 2 \pi$ (i.e. an estimate of the average peak velocity) is $\bar{u}_{p} / U_{0}=0.46$ with a standard deviation of $\sigma_{p} / U_{0}=0.03$. 